OPEN ACCESS

Edited by:

Fabio Benfenati,

Istituto Italiano di Tecnologia, Italy

Reviewed by:

Carlos Vicario-Abejón,

Spanish National Research Council

(CSIC), Spain

Susanna Bosi,

University of Trieste, Italy

*Correspondence:

Qi Zhang

zhangq@health.fau.edu

Received: 30 September 2018

Accepted: 24 June 2019

Published: 16 July 2019

Citation:

Kitko KE and Zhang Q

(2019) Graphene-Based

Nanomaterials: From Production to Integration With Modern Tools in

Neuroscience.

Front. Syst. Neurosci. 13:26. doi: 10.3389/fnsys.2019.00026

\section{Graphene-Based Nanomaterials: From Production to Integration With Modern Tools in Neuroscience}

\author{
Kristina E. Kitko ${ }^{1,2}$ and Qi Zhang ${ }^{3 *}$ \\ ${ }^{1}$ Program in Interdisciplinary Materials Science, Vanderbilt University, Nashville, TN, United States, ${ }^{2}$ Department \\ of Pharmacology, Vanderbilt University, Nashville, TN, United States, ${ }^{3}$ The Brain Institute, Florida Atlantic University, \\ Jupiter, FL, United States
}

Graphene, a two-dimensional carbon crystal, has emerged as a promising material for sensing and modulating neuronal activity in vitro and in vivo. In this review, we provide a primer for how manufacturing processes to produce graphene and graphene oxide result in materials properties that may be tailored for a variety of applications. We further discuss how graphene may be composited with other bio-compatible materials of interest to make novel hybrid complexes with desired characteristics for bio-interfacing. We then highlight graphene's ever-widen utility and unique properties that may in the future be multiplexed for cross-modal modulation or interrogation of neuronal network. As the biological effects of graphene are still an area of active investigation, we discuss recent development, with special focus on how surface coatings and surface properties of graphene are relevant to its biological effects. We discuss studies conducted in both non-murine and murine systems, and emphasize the preclinical aspect of graphene's potential without undermining its tangible clinical implementation.

Keywords: graphene, graphene oxide, neuron, plasma membrane, cytotoxicity, synapse, neurotransmission

\section{INTRODUCTION}

The promises of nanomedicine are extensive. Graphene (Gr), the first true two-dimensional material to exist in isolation, is the type of new nanomaterial that results in interest for novel biomedical applications. From Michael Chrichton's tragic protagonist in The Terminal Man to the recent growth in start-up companies seeking to transfer consciousness, the fictive present and future call to mind visions of devices that enable neural interfacing and control. Although these ideas may create questions as to ethics for neuroscience in the future, the current state-ofthe-art for implanted devices is far more limited in scope. Progress in brain-computer interfaces holds great promise for patients following stroke (Ramos-Murguialday et al., 2013), to control prosthetic limbs (Hochberg et al., 2006; Donoghue et al., 2007), with the motor degeneration characteristic of Parkinson's disease (Little et al., 2013), and for a variety of other disorders and diseases (Chaudhary et al., 2016). Gr may be poised for incorporation into such devices. 
As the presence of $\mathrm{Gr}$ becomes more widespread and commonplace across the biomedical sciences, the relatively larger body of work detailing the biological effects of carbon nanotubes may serve as a template guiding the utility of $\mathrm{Gr}$ for biological applications (Kostarelos et al., 2009).

Gr-based materials for interfacing with the peripheral nervous system have been reviewed elsewhere (Domínguez-Bajo et al., 2017; Bramini et al., 2018). We instead focus on new directions for application to the central nervous system. This review is limited to preclinical applications, although Gr and Gr-based devices may someday advance to clinical implementation. We begin with an overview of $\mathrm{Gr}$ manufacturing advances, applications to hybrid materials systems as well as drug delivery strategies. This is followed by an overview of work performed with non-murine models. Finally, the interaction of $\mathrm{Gr}$ with murine neural systems, both in vivo and in vitro is examined. Despite a sizable body of work, to date, there remain many unresolved questions as to cytotoxicity and the mechanisms underlying the Gr-cellular interaction that must be addressed moving forward.

\section{MANUFACTURING PROCESSES}

2D graphite was long believed to be relegated to the realm of theoreticians and condensed matter physicists, as the thermodynamic stability of such crystals was believed to be prohibitive for their existence (Geim and Novoselov, 2007). 2D graphite - or "graphene" - was first isolated through mechanical exfoliation (Novoselov et al., 2004, 2005). These small sheets provided the ability to study transport in this new class of material (Novoselov et al., 2004), but the small size of the sheets $(<10 \mu \mathrm{m})$ necessitated the development of alternative approaches that would produce $\mathrm{Gr}$ in sizes large enough for practical transistor-based applications. Of note, for high quality single and few layer $\mathrm{Gr}$ sheets, mechanical exfoliation remains the process of choice for transport measurements to date. However, an array of production methods are now enabling production of high-quality $\mathrm{Gr}$ in ever higher qualities and ever larger areas (Figure 1).

The development of chemical-vapor deposition as a manufacturing strategy (Li et al., 2009a; Reina et al., 2009) allows the production of $\mathrm{Gr}$ with higher area coverage than was previously possible with exfoliation-based methods. To date, the majority of devices that demonstrate compatibility for in vivo imaging use single or multi-layer Gr produced via chemical vapor deposition (Kuzum et al., 2014; Park et al., 2014, 2016, 2018; Du et al., 2015). Indeed, advances in CVD technology have allowed the production of rectangular $\mathrm{Gr}$ sheets with a cross-length as long as 30 inches (Bae et al., 2010). Although these large size sheets should be of sufficient size for any neural application, the solution transfer process often results in alteration of the properties of the Gr sheet (Suk et al., 2011, 2013). Thus, continued research into scalable transfer of CVD Gr or alternative processes (Pang et al., 2017) should help generate more reliably responsive devices. Additionally, ensuring that devices are processed in such a way as to remain relatively sterile is a necessary step to consider for long-term interfacing.
As an alternative to the large-scale growth of $\mathrm{Gr}$, it is now possible to produce large quantities of single-layer and multilayer Gr through bulk exfoliation (Hernandez et al., 2008; Li et al., 2008; Lotya et al., 2009; Shih et al., 2011; Paton et al., 2014). Graphite in a colloidal suspension can be sonicated to yield thin Gr flakes, ranging from single to a few layers. A variety of solvents are compatible with this approach-and more recently it has been demonstrated that $\mathrm{Gr}$ and $\mathrm{Gr}$ oxide (GO) can be exfoliated directly into biological media (Castagnola et al., 2018). GO flakes produced by this method are more largely monolayer than $\mathrm{Gr}$ flakes, however, oxidization comes with costs, for example, reduced carrier mobility. Flakes of Gr produced by exfoliation, while not matching the transport properties of micromechanical isolation, can be considered Gr, as no chemical functionalization is required. Thus, Gr flakes may prove advantageous for applications where the electronic properties are not of primary importance but where large quantities of $\mathrm{Gr}$ are desirable. To be noted, long-term stability of Gr flake suspension still requires coating with surfactant as the hydrophobic surface of those flakes readily forcing them to form aggregates (unpublished result).

The ease with which GO can be chemically modified remains of interest for drug delivery and bio-scaffolding applications. A number of different processes have been developed to produce bulk quantities of GO flakes (Brodie, 1860; Hummers and Offeman, 1958), although GO is traditionally produced through the reduction of bulk graphite in the presence of both acids and oxidants (Park and Ruoff, 2009). Reduction of GO yields Gr-like sheets ( $\mathrm{rGO}$ ) with improved electrical conductivity (Stankovich et al., 2007), but the electronic properties of rGO still lag far behind those of pure Gr, even with the numerous efforts that have been made via modifying annealing processes to improve the figures of merit. The inherently lower electrical conductivity and inability to greatly increase it has resulted in less interest to date in the use of GO as an electrode material. Indeed, even chemical reduction of GO to rGO yields room temperature conductivity values three orders of magnitude below that measured for pristine Gr (Gómez-Navarro et al., 2007). Mobility values for GO produced via the Hummers method are $\sim 850 \mathrm{~cm}^{2} /(\mathrm{V} \mathrm{s})$, but other methods report a device mobility of around $\sim 1-10 \mathrm{~cm}^{2} /(\mathrm{V} \mathrm{s})$ (Eda et al., 2008; Wang et al., 2008; Su et al., 2009). Importantly, future device design may take into account contact resistance in production, as use of all-carbon transistors improve both electron and hole mobility relative to using gold electrodes (Wang et al., 2010), which may prove a more practical approach to improve device performance. Further attempts to reduce GO to Gr-like sheets via chemical (Li et al., 2009b; Moon et al., 2010) or thermal (Becerril et al., 2008; Jung et al., 2009; Barroso-Bujans et al., 2010) processing have improved the fraction of graphitic areas in the structure, but also introduced nanoscale holes and defects that deleteriously affect final performance. Ultimately, for practical application, if devices are to be manufactured at a commercial scale, inconsistencies introduced in such devices will have to be carefully characterized to ensure that recorded signals are representative of neural responses. rGO's improved device characteristics also come at the cost of a reduction in hydrophilicity (Rourke et al., 

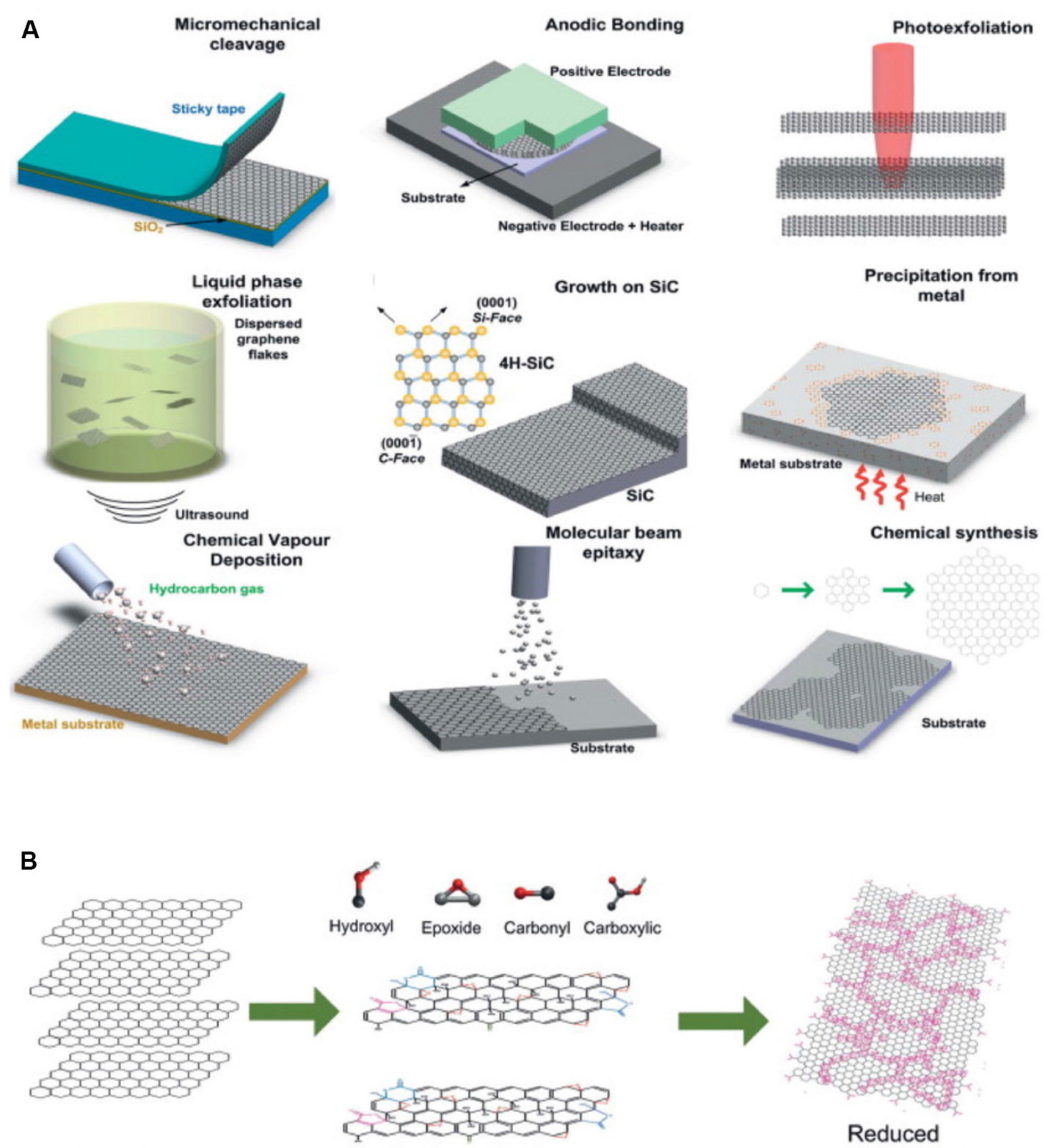

Graphite

Graphene Oxide

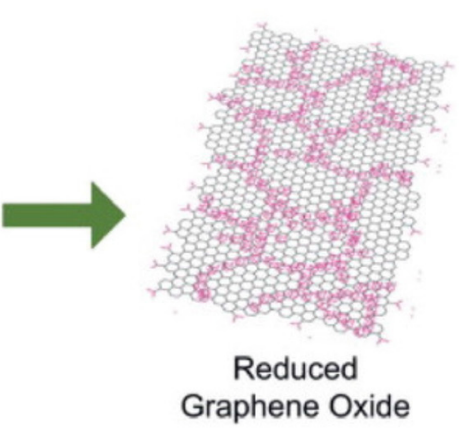

FIGURE 1 | Production of Graphene/Gr oxide (Gr/GO). (A) Gr production methods and (B) GO production and reduction methods. Modified with permission from Bonaccorso et al. (2012).

2011), and surface properties remain an important consideration for bio-interfacing applications. It was recently demonstrated that solution-exfoliated GO flakes could be processed to recover properties more resembling those of CVD-produced Gr. Reduction of the concentration of in-plane oxygen via 1-2 s microwave pulses produced $2 \mathrm{D}$ and $\mathrm{G}$ peaks closer to those of Gr and greatly increased electron and hole carrier mobility to $\sim 1,000 \mathrm{~cm}^{2} /(\mathrm{V} \mathrm{s}$ ) in a FET (Voiry et al., 2016). Thus, the ability to modify in-plane oxygen concentration to improve electronic properties may open new doors to the use of GO and GO-based materials for sensor applications.
Similar to applications for the photoluminescence of CNTs (Welsher et al., 2008), the photoluminescence of GO may be useful in for optical readout and/or in combination with drug delivery. The photoluminescence of GO, which arises from bond disorder throughout the structure, which induces energy gaps (Cao et al., 2013), is to some extent a tunable property, depending on the oxidization state (Luo et al., 2009). This stands in contrast to the lack of photoluminescent emission observed for defect-free Gr. GO luminescence is broadband in as-prepared samples, with a wide peak across the visible spectrum (Luo et al., 2009; Qian et al., 2012). PEGylated GO sheets exhibit intrinsic 
near-infrared red Hernandez et al., 2008 photoluminescence (Sun et al., 2008), a property of great interest for in vivo imaging applications due to the enhanced light penetration in this wavelength range. It has been more recently demonstrated that $\mathrm{GO}$ also exhibits photoluminescence under both two-photon and three-photon excitation (Qian et al., 2012), in addition to the broad photoluminescence in the visible range. By exploiting the ability of GO to undergo a two-photon process, Qian et al. (2012) imaged PEGylated-GO nanoparticles in a skull-removed whole brain to a depth of $300 \mu \mathrm{m}$. Growth in the commercial availability of three-photon sources may lead to studies at even greater depths within the intact rodent brain. As new advances in genetic engineering and microscopy enable deeper and faster cellular resolution imaging in head fixed or freely moving specimens, it is likely GO/Gr-based imaging will also move toward applications compatible with this type of experiment. Although the broadband nature of the emission from GO may be somewhat of a limiting factor for multiplexing multiphoton imaging processes, advances in hyperspectral detection and fast fluorescence lifetime detection may help to make GO of greater utility for in vivo brain imaging.

\section{GRAPHENE/POLYMER COMPOSITE MATERIALS AND APPLICATIONS}

Polymer electronics remain of great interest to ultimately offer an alternative to traditional materials to minimize mechanical mismatch between cells/tissue and the recording probe (for a more in-depth discussion see Rivnay et al., 2017). Elastic modulus mismatch between brain tissue and recording device leads to increased tissue damage both upon insertion and during chronic interface (Polikov et al., 2005), as the elastic moduli of brain tissue $(\sim 150 \mathrm{kPa})$ and an implanted electrode $(\sim 150 \mathrm{GPa}$ for silicon) differ by six orders of magnitude. Thus, much current research has focused on the ability to better match the modulus of electrodes to that of brain tissue (for a more in-depth review on interfacing tissue with electrodes, see Fattahi et al., 2014). Gr electrodes have been of interest for such designs, as it can be incorporated into flexible electronics (Fiori et al., 2014); and for applications in the brain, where techniques like optogenetics and calcium imaging require optical access to brain regions of interest, the large degree of optical transparency of single or few-layer Gr (FLG) may be uniquely advantageous.

\section{PEDOT}

Poly-3, 4-ethylene dioxythiophene (PEDOT) is an electroconductive polymer produced from 3, 4-ethylene dioxythiophene monomers. Polymerization results in a positively-charged backbone, whereby negatively charged materials can then be incorporated to balance charge. Neurons embedded in PEDOT matrices remain viable for around 1 week (Richardson-Burns et al., 2007) and neurons grown on PEDOT-based substrates show unaltered electrophysiological characteristics (membrane potential, membrane capacitance, input resistance) after 21 DIV (Cellot et al., 2016). Although the surface charge of Gr limits its utility in PEDOT-based composites without further surface modification, the negative surface charge of $\mathrm{GO}$ may be better adaptable to such composites. PEDOT/GO composites have been used as electrode coatings to improve sensitivity and decrease the lower detection limit of dopamine in fast-scan cyclic voltammetry (Taylor et al., 2017), a widely-used technique for measuring dopamine release in rodents in vivo (Robinson et al., 2003). Carbon nanotube-PEDOT composites have also been demonstrated to perform well in interfacing applications (Jan et al., 2009; Luo et al., 2011). PEDOT-coated microelectrode arrays show good performance characteristics and lowered impedance relative to iridium oxide ( $\mathrm{IrOx}$ ), suggesting a potential for long-term neural interfacing applications (Wilks et al., 2009). As both GO and CNT incorporation into PEDOT-based composites has improved overall performance, future work may seek to fabricate composite polymer electrodes.

\section{Chitosan}

Chitosan composites have been demonstrated both for GO (Yang et al., 2010; Bao et al., 2011) and for rGO (Fang et al., 2010). It can be produced in relatively abundant quantities from the deacetylation of chitin. Like with many polymers, applications may be limited by the low mechanical strength of the material. GO as a nanofiller is one route to achieve enhanced mechanical properties. Chitosan-GO nanocomposites can be assembled in a manner of ways. $\mathrm{pH}$-responsive functionality is possible with chitosan (Yi et al., 2005); increased $\mathrm{pH}$ leads to amine deprotonation, decharging of the polymer, and ultimately insolubility. Interestingly, preparations of such suspensions seem to be greatly affected by the preparation method: addition of GO to chitosan yields a uniform suspension while addition of chitosan to GO yields agglomerations (Fang et al., 2010). This is due to the way that excess GO will create bridges between sheets via multiple attachment points on the polymer chains. Reducing GO allows chitosan attachment by zwitterionic interactions and hydrogen bonding between the remaining oxygen groups of the rGO and the amino and hydroxyl groups of chitosan. The reversibility of the molecular chain interactions with GO sheets between different $\mathrm{pH}$ values may provide an opportunity to modulate Gr-based composite materials within different cellular compartments. This could potentially allow for pH-based assembly strategies in acidic intracellular compartments (e.g., late endosomes, lysosomes), where chitosan would stabilize $\mathrm{GO}$ composites relative to higher $\mathrm{pH}$ extracellular spaces.

\section{Hydrogels}

The use of Gr for regenerative approaches has been reviewed previously (Ding et al., 2015), however the pace of new methodologies in neuroscience has opened new directions for scaffolding technologies, with a particular resurgence in hydrogel-based techniques for connectome applications (Chung et al., 2013; Chen et al., 2015). Gr/GO and other nanomaterials may be of interest for cleared or expanded tissue applications where added structural stability is desirable. Composite scaffolds for regenerative medicine remain and area of great interest. 
Hydrogels can be chemically tuned to impart different surface properties, for example, to modify surface charge or conductivity, before functionalization to GO (Liu X. et al., 2017). Biomolecules such as DNA can also be incorporated via stacking interactions (Xu et al., 2010), enabling payload delivery within the hydrogel matrix. This is one possible route to achieve more biologically realistic synthetic minimal brain circuits, which to date have largely been limited in structure to two-dimensional culture systems or proteins or liposomes (Adamala et al., 2017). Overall mechanical strength can be tailored by the degree to which $\mathrm{Gr} / \mathrm{GO}$ is incorporated, with mechanical strength being inversely correlated to the amount of swelling in the composite. Local delivery of polymerized materials may someday be a route to a new form of tissue scaffolding in vivo. In such applications, Gr and Gr-based materials may play a multifunctional role, both as structural support and as part of a stimulation or recording device.

\section{Graphene in "Stretchable" Electronics Applications}

Advances in both computational and analytical models have recently begun to enable the fabrication of nanoscale semiconductor materials that will tolerate relatively large amounts of strain (Su et al., 2017; Yu et al., 2017), advances in the manufacturing of Gr may allow similar structures to be produced (Wang et al., 2017). Although measurements of second-order stiffness in graphene have yielded in-plane stiffness values of $\sim 340 \mathrm{~N} / \mathrm{m}$ (Lee et al., 2008), crumpling from static wrinkling in free-standing $\mathrm{Gr}$ at biologically relevant temperatures effectively reduces this value (Nicholl et al., 2015). p-type doping of Gr may be one way forward for flexible $\mathrm{Gr}$ electrodes, as it decreases sheet resistance and increases the effective work function (Han et al., 2016). Multilayer-based approaches using Gr may also improve stretchability performance through strain relaxation (Won et al., 2014). In fact, the addition of Gr "nanoscrolls" between layers in transparent transistors showed improved performance under strain relative to monolayer Gr (Liu N. et al., 2017); and multilayer composite or flexible devices must be designed with consideration to the properties of Gr/GO that are being utilized. For example, PEDOT electrodes with sufficient recording capability where $\mathrm{Gr}$ may be incorporated to increase overall mechanical strength may have different design criteria from applications where Gr acts as an electrode material.

\section{DRUG DELIVERY APPLICATIONS AND CONJUGATION STRATEGIES}

\section{Chemical Modifications for Drug Delivery Applications}

Gr/GO have been most widely demonstrated for cancer-related drug delivery applications (Liu et al., 2011; Liu C.-C. et al., 2017), however, the chemical and surface modifications used to enable loading and release may also be used to enable new applications in the brain. The ability to harness hydrophobic interactions and $\pi-\pi$ stacking to deliver aromatic, hydrophobic compounds may extend the utility of Gr for brain-specific drug delivery beyond simply proof-of-concept. For example, polyethylene glycol modification (PEGylation) of GO results in excellent solution stability (Liu et al., 2008). Alternative strategies also include Polyamidoamine (PAMAM) functionalization of both $\mathrm{GO}(\mathrm{Gu}$ et al., 2017) and Gr (Quintana et al., 2011) and hyperbranched polyglycerol (hPG; Tu et al., 2017). Amide linkage between GO and chitosan yields sheets that are relatively stable in cell culture media for up to $48 \mathrm{~h}$ (Bao et al., 2011), an example of a myriad of alternative approaches to stabilize $\mathrm{Gr} / \mathrm{GO}$ in aqueous solutions. Dextran can also be used to increase the hydrophilicity of GO via amine modification and EDC coupling chemistry (Zhang et al., 2011). GO functionalized with cyclodextrin molecules, again via $\pi-\pi$ adsorption, reduced to Gr sheets in an ammonia solution also serves as an effective peptide carrier (Dong et al., 2013). Thus, the versatility of $\pi-\pi$ adsorption onto $\mathrm{Gr} / \mathrm{GO}$ surfaces allows for a wide scope of possible molecular delivery types.

Chemical reaction methods can also be selected to control the location of functional groups onto $\mathrm{Gr}$ sheets. 1,3-dipolar cycloaddition results in conjugation within the large central area of the sheets, whereas amide concentration reactions concentrate conjugates to the edges of Gr sheets (Quintana et al., 2011). Azide modified dopamine has been used for simultaneous capping and reduction of GO (Kaminska et al., 2012), where the aromatic structure of the dopamine molecule likely interacts via $\pi-\pi$ stacking on the surface. As many monoamines contain aromatic groups, molecules such as serotonin, cathecholamine, and epinephrine may find utility as stabilizers for GO while also acting to alter neural function. Although click chemistry opens new doors to functionalization strategies for $\mathrm{Gr} / \mathrm{GO}$, approaches that utilize a copper catalyst elicit concern regarding toxicity to living tissue (Baskin et al., 2007).

Interestingly, the overall surface charge of a GO sheet was shown to play a role in the effectiveness of intracellular drug delivery. Positively-charged aminolated surfaces were shown to be more effective at releasing Doxorubicin (DOX) in intracellular compartments than negatively charged sulfonated surfaces $(\mathrm{Tu}$ et al., 2017). Given the relative ease of modifying the surface charge of GO, this may be a new avenue to site-specific intracellular drug release. The different surface characteristics across a Gr sheet may also be a useful strategy for orthogonal delivery of different classes of compound: the negatively charged surface regions may better adsorb positively charged molecules while the outer edges, decorated with carboxyl groups, can, for example, be modified with zwitterionic lipid vesicles (Wang et al., 2013).

\section{Payload Delivery}

The delivery of various forms of genetic payload has been demonstrated as a possible application for Gr/GO-based materials, however, to date, low transduction efficiencies limit the utility of $\mathrm{Gr}$ in comparison to traditional methods for genetic delivery. For example, chitosan-stabilized GO sheets had a lower transfection efficiency for luciferase transduction into HeLa cells (Bao et al., 2011) compared to traditional methods. Polymerbased assemblies are some of the most widely used nanomaterial strategies for transduction, and relative to polymer-based 
approaches, GO used to attach plasmid DNA and PEI shows improved transfection efficiency (Feng et al., 2011). Gr can also bind ssDNA, although cannot bind dsDNA to the same extent. This property has been exploited to deliver hairpin-shaped DNA into cells, which will be unloaded upon interaction with an mRNA target (Lu et al., 2010). GO has also been employed as a delivery vehicle for aptamers, delivering an ATP-binding aptamer (Wang et al., 2010) to cells. Gr/GO may ultimately be most advantageous for applications where simultaneous delivery of both genetic payloads and pharmacological compounds is desirable. For example, PAMAM functionalized GO was demonstrated as a vehicle for both DOX and shRNA (Gu et al., 2017) delivery.

\section{Photothermal Therapy}

Reduced GO (rGO) has been exploited for its photothermal properties for drug delivery. Chitosan/rGO composites were shown to deliver drug payloads on a timescale of minutes; addition of rGO to Chitosan acts to increase the photothermal absorption of the composite with respect to chitosan alone (Matteini et al., 2014). Here, DOX delivery to HeLa cells was increased with short-pulse laser illumination. DOX has also been loaded onto GO for photothermal delivery in a glioma-bearing rat model (Liu et al., 2013; Dong et al., 2016). Laser irradiation results in local surface heating, ultimately leading to drug release. DOX release was also demonstrated to be effective on gliomas when loaded on PEGylated silica-coated Gr sheets (Wang et al., 2013). Carboxy-modified GO was covalently linked to Thioflavin $S$, which made it selectively attached to amyloid- $\beta$ fibrils (Li et al., 2012), a potential avenue toward the photothermal dissociation of $\mathrm{A} \beta$ fibrils and demonstrating the potential of $\mathrm{Gr} / \mathrm{GO}$-based materials for therapeutic application to Alzheimer's disease. Hydrazine reduction of GO at elevated temperatures increases NIR absorbance by $>6$-fold relative to unreduced GO (Robinson et al., 2011), a function of the restoration of $\pi$ conjugation. In the most widely used state, largely due to the relative ease of production and low cost, Gr flakes exist as a semimetal with zero bandgap. More recently, the discovery that a "nanomesh" structure can open up a bandgap in Gr (Akhavan, 2010; Bai et al., 2010) can be used to tune photothermal absorption properties. PEGylated rGO nanomesh suspension showed a much steeper temperature increase over time for NIR irradiation heating than PEGylated rGO (Akhavan and Ghaderi, 2013). Although CNTs have been more widely used for photothermal therapy to date, the superior response of Gr (Markovic et al., 2011) may lead to increased focus in this direction.

Laser irradiation with NIR light enables a relatively high degree of spatial precision. However, for in vivo applications, the ability to control release will ultimately be limited by the ability to deliver light within the brain. As such, NIR will be a useful tool for fundamental studies, as differential effects between even superficial sub-regions within the brain are still not well understood. Alternative triggering methods may be better suited to study where pharmaceutical effects are elicited in deep brain regions. Electrical, magnetic, or even acoustic-based triggered release would allow such control in deeper brain regions. Layerby-layer assembly approaches utilize protein adsorption onto substrates and subsequent capping with modified GO in either a sheet (Hong et al., 2012) or a capsule (Kurapati and Raichur, 2012) format. Additional layers can be stacked together to control overall release time (Hong et al., 2012). Passive release may be sufficient for the delivery of certain drug classes, but active release allows more precise control of treatment dose received. PAE (Choi et al., 2015) or PPy (Weaver et al., 2014) films can incorporate GO, resulting in a more conductive polymer matrix, whereby electrical stimulation is applied and elicits drug release. Modification of the number of GO layers and the overall areal size of the GO sheets also alters the total drug loading capability (Weaver et al., 2014); smaller and fewer layered sheets have increased surface area for adsorption relative to more multilayered stacks. Photothermal irradiation has also been utilized to target delivery to cytosolic locations. Although $\mathrm{Gr} / \mathrm{GO}$ sheets can insert into membranes directly, small sized and few-layered sheets will also be taken up into the cell through endocytic processes. Ultimately these sheets will then be trafficked to endosomal compartments. rGO sheets have been used to help payload escape this fate by application of NIR irradiation to induce endosomal disruption (Kim et al., 2013).

\section{Magnetic Applications}

The ability to modify the properties of $\mathrm{Gr} / \mathrm{GO}$, to confer magnetic sensitivity for example, will extend the utility of its applications. The presence of fluorine in the GO basal plane can induce paramagnetic centers, making fluorinated-GO compatible with MRI applications (Romero-Aburto et al., 2013). Magnetic nanoparticles such as iron oxide $\left(\mathrm{Fe}_{3} \mathrm{O}_{4}\right)$ can also be loaded on the surface of GO, conferring sufficient contrast enhancement to enable MRI (Yang et al., 2013). This does not disrupt the ability of $\mathrm{Gr} / \mathrm{GO}$ to act as a drug delivery vehicle, further extending its utility.

\section{TOXICITY IN MURINE SYSTEMS}

The extent to which $\mathrm{Gr} / \mathrm{GO}$ become practically applicable to neuroscience will in part be determined following a systematic understanding of long-term toxicity. As many paths toward clinical application begin with pre-clinical testing in murine models, understanding the biological tolerance of rats and mice to $\mathrm{Gr} / \mathrm{GO}$ represents an important first step. Here, we focus on toxicity specifically relevant to neuronal and brain-wide function, for discussion of overall, environmental, or antimicrobial toxicity, which have been widely reviewed, see elsewhere (Seabra et al., 2014).

Various studies have also focused on the interaction between $\mathrm{Gr}$ and the cell membrane in either in vitro culture systems (Kitko et al., 2018) or lipid bilayer preparations. Using a two-dimensional Langmuir-Blodgett approach, it has been suggested that the hydrophobic tail of lipids does not play a role in any bilayer interactions, but a positively charged head group would favor interactions with the carboxy-containing regions of GO (Li et al., 2013) and minimal interactions would occur between neutrally or negatively charged lipids. The size of the $\mathrm{Gr} / \mathrm{GO}$ flakes is also a determining factor in the bilayer response. Flakes of GO that are large relative to the size of 
an artificial liposome cause rupture of the bilayer attached to a substrate surface (Frost et al., 2012). Addition of GO to supported lipid bilayers (SLBs) composed of DPPC/DOPC causes detachment of bilayer regions (Lei et al., 2014), but as this was a function of relatively high levels of calcium used in SLB preparation, may not be viewed as representative of in vivo membrane damage.

The results based on computational modeling generally agree that once inside of a lipid bilayer, either via endocytic uptake or by direct membrane penetration, Gr will stably reside between phospholipid tails (Titov et al., 2010; Guo et al., 2013). It is generally agreed that membrane penetration would favor an "edge-in" rather than a "face-in" initial contact.

Although computational models are powerful tools to provide fundamental insights into the forces governing $\mathrm{Gr} / \mathrm{GO}$ nanomaterial-cellular interactions, these studies are often performed under conditions necessitated by restrictions on the ability to model different membrane components, for example, protein coronas and extracellular-matrix components secreted by neurons are largely not included in such models. It is furthermore prohibitive to model the membrane bilayer with the full complexity of proteins, lipids, and other molecules within a neuronal bilayer. In addition, it is computationally prohibitive to model $\mathrm{Gr} / \mathrm{GO}$ flakes on the same size scales that are produced for experimental studies. A "large Gr/GO flake" in a computational study may be on the length scale of $5 \mathrm{~nm}$ (Li et al., 2013)-whereas for experimental studies the smallest reported average dimensions are on a length scale of $>200 \mathrm{~nm}$ (Rauti et al., 2016; Castagnola et al., 2018). Thus, it may be difficult to draw direct parallels between toxicity claims from simulations and toxicity claims from experimental results. Study of lipid-membrane specific effects is more efficiently enabled by allowing $\mathrm{Gr}$ to penetrate a membrane after addition to biological media. However, many studies, even using small flakes of $\mathrm{Gr} / \mathrm{GO}$, deposit the material onto a glass substrate for chronic cellular interface. The membrane interactions here would be very different from $\mathrm{Gr} / \mathrm{GO}$ located within the lipid bilayer, further complicating arguments as to the membrane effects of $\mathrm{Gr} / \mathrm{GO}$; and same caution should be paid to the bare or surfactantcoated $\mathrm{Gr}$ as the former will aggregate and result in different physicochemical properties.

GO was demonstrated to be toxic to gram-negative bacteria (Akhavan and Ghaderi, 2010; Tu et al., 2013), yet bacteria containing more complex outer membranes are more resistant to damage. Reduction of GO ( $\mathrm{rGO}$ ) increases susceptibility to membrane damage (Akhavan and Ghaderi, 2010). Akhavan (2010) fabricated nanowalls of GO, which were designed such that there would be a maximal amount of direct contact between bacteria and the sharp edges of the nanomaterial. This represents a condition that would induce mechanical stress on the membrane and indeed can result in membrane damage. Tu et al. (2013) later extended this work both through molecular dynamics simulations and experiments using E. Coli. Coursegrained molecular dynamic simulations of relatively large FLG sheets suggest that the most hydrophobic edge of Gr near a lipid bilayer will penetrate orthogonal to a bilayer, and then fully embed in a membrane, driven by an attraction between the $\mathrm{Gr}$ and lipids within the core (Li et al., 2013). Interestingly, this spontaneous process does not result in membrane destruction, suggesting that the degree of mechanical stress on the membrane plays a role in membrane damage when exposed to $\mathrm{Gr}$ or GO. That spontaneous membrane incorporation does cause membrane destruction is in agreement with experimental observations using cultured PC-12 cells, where 24-h exposure to FLG sheets did not increase lactate dehydrogenase activity or increase reactive oxygen species below $100 \mu \mathrm{g} / \mathrm{mL}$ treatment concentrations (Zhang et al., 2010).

Of note, these configurations, where membrane stress is likely a factor in the toxicity of $\mathrm{Gr} / \mathrm{GO}$, are different than most studies to date using neuronal cultures, where $\mathrm{Gr} / \mathrm{GO}$ is more commonly used as a culture substrate. Three different mammalian cell types cultured on rGO, but not GO, for up to 5 days proliferated normally and exhibited less cytotoxicity and more outgrowth than on CNT films (Agarwal et al., 2010). HT-29 cells also displayed increased attachment on GO-coated substrates within $6 \mathrm{~h}$ compared to bare glass substrates (Ruiz et al., 2011). Thus, future studies aimed at addressing nanotoxicity would benefit from drawing a distinction between scenarios where mechanical stress may be an additional factor and scenarios where spontaneous membrane incorporation alone is being studied.

Simulations also suggest that the hydrophobicity of $\mathrm{Gr} / \mathrm{GO}$ plays a role in its interaction with the bilayer and that the surface energy can be modified by the formation of a protein corona on the surface. For example, computational models demonstrate that the presence of a protein corona surrounding the flakes would modify the membrane response to $\mathrm{Gr}$, in which case Gr would orient in parallel to and attach to the outer layer of the lipid bilayer (Li et al., 2013). Experimental studies of protein adsorption on $\mathrm{Gr} / \mathrm{GO}$ alone have shown that nanoflakes can adsorb $1.6-2 \times$ their weight in BSA, largely on the timescale of minutes (Hu et al., 2011; Chong et al., 2015). A recent development, where Gr is exfoliated directly into a serum containing media, has given some insight into the composition of the biological corona formed. Proteomic analysis of these media-exfoliated Gr flakes reveals a variety of proteins and other cellular materials that make up the protein corona formed on Gr (Castagnola et al., 2018): serum albumin, apolipoproteins, and vitronectin are all found on Gr nanoflakes in relatively large abundances. Although the physicochemical interactions, kinetics, and thermodynamic processes that govern the formation and evolution of the corona that forms around nanomaterial surfaces when interfaced with a biological system is still not fully understood, general frameworks have been established as to the governing forces underlying nano-bio interactions. Because the individual environment Gr/GO encounter will vary widely depending upon desired neural application, the exact composition of the corona formed cannot necessarily be described a priori or results extended from one biological system to the next. Indeed, the chemical concentration, surface functionalization, degree of crystallinity, and surface roughness, among many properties, all play a role in the composition and evolution of biomaterials that adsorb on a nanomaterial surface (Nel et al., 2009). 
The formation of a protein corona on $\mathrm{Gr} / \mathrm{GO}$ surfaces also differs between bacterial cultures and what would be observed in murine models in vivo due to differences between media compositions in cell culture or fluid composition in the extracellular space. Previous studies demonstrating membrane destruction in bacteria have suspended Gr flakes with E.Coli (Tu et al., 2013) or suspended in agar/water and dropped onto a substrate surface and later recovered (Akhavan and Ghaderi, 2010). However, the presence of a protein corona (Cedervall et al., 2007) likely plays a role in mitigating these effects. Using A549 cells, multiple reports have demonstrated that the presence of FBS in normal culture media (Hu et al., 2011; Duan et al., 2015) or the addition of BSA (Li et al., 2014; Duan et al., 2015) result in lower cytotoxicity of GO flakes relative to serum-free media. Using a DPPC model membrane, molecular dynamics simulations revealed that $\mathrm{BSA}$-coating of $\mathrm{Gr}$ reduces the total amount of lipid removal relative to bare $\mathrm{Gr}$. The coating of $\mathrm{Gr}$ by proteins is governed by hydrophobic interactions, van der Waals forces, and $\pi-\pi$ interactions (Chong et al., 2015). The composition of the protein corona may vary depending upon the method of introduction to the brain and the presence of any surface modifications to increase biocompatibility; this may also serve to explain the variation in effects seemingly exerted by $\mathrm{Gr}$ (Radic et al., 2013). As the adsorption of proteins to GO is strong and long-lasting, this may serve as one route for the low-cost modification of GO for drug delivery application or to achieve loss or gain of function cellular control in some manner (Belling et al., 2016).

Although many studies that attempt to evaluate the effects of Gr may lessen the extent to which a protein corona is involved by incubation in serum free-media (Zhang et al., 2010; Pampaloni et al., 2018), the relatively long exposure times used may still result in the coating of nanomaterial surfaces by excreted proteins in cell culture. Indeed, proposed mechanisms for the effects underlying chronic culture on graphene have suggested that Gr still plays a large and direct mediatory role, rather than an indirect through a protein corona (Kitko et al., 2018; Pampaloni et al., 2018). Understanding the role of cell secretion in mediating the toxicity of Gr-based materials remains an important line of future investigation.

Surface functionalization has also been shown to play an important role in the toxicity of Gr/GO. For example, PEGylation decreases the overall cytotoxicity of GO (Li et al., 2014). And bilayer Gr functionalized with carboxyl groups showed improved viability in kidney cells relative to pristine bilayer $\mathrm{Gr}$ at concentrations above $\sim 5 \mathrm{mg} / \mathrm{L}$ (Sasidharan et al., 2011). $\mathrm{Tu}$ et al. (2013) modified GO with $-\mathrm{OCH}_{3},-\mathrm{NH}_{2}$, or -PABS via PEGylated chains and cultured hippocampal neurons to 7 DIV to determine the effect of surface charge on neuronal viability and outgrowth. PEG-amine modified GO exhibited the most positive surface charge and the most neuronal outgrowth relative to other surface treatments or the native- $\mathrm{COOH}$ group (Tu et al., 2014), suggesting the importance of the surface in determining neuronal responses to GO. This is in line with what was observed for mouse hippocampal neurons on CVD-Gr substrates, where pristine $\mathrm{Gr}$ was shown to improve viability and connectivity up to $5 \mathrm{DIV}$, whereas disordered noncrystalline Gr did not result in any neuronal attachment (Veliev et al., 2016). Thus, the crystallinity of $\mathrm{Gr}$ is also an important consideration in evaluating neuronal responses. Given the overall inconsistency among assessments of nanotoxicity for $\mathrm{Gr} / \mathrm{GO}$, it is likely that surface charge plays a role. Given the number of fabrication methods, transfer processes, and application methods for these materials, comprehensive studies of toxicity should include measurement and reporting of relevant surface characteristics (Faria et al., 2018).

\section{DISTRIBUTION AND TRAFFICKING OF Gr/GO IN VIVO}

Mass spectrometry is a common approach to determine the bio-distribution of nanomaterials. For Gr/GO, most distribution studies to date have focused on overall distribution following tail vein injection, with results indicating that very little trafficking to the brain will happen via this route. MALDI-TOF was used to determine that tail vein injection of GO results in very little accumulation in the brain after $24 \mathrm{~h}$ (Chen et al., 2015), in good agreement with what was observed using radiolabeled GO for similar time periods (Zhang et al., 2011). Tail vein studies performed using rGO indicate uptake in the brain within $15 \mathrm{~min}$ for tail vein injection, peaking around $3 \mathrm{~h}$ and decreasing by 7 days, which was corroborated by confocal microscopy (Mendonça et al., 2015). This is somewhat surprising given the relatively large-sized flakes used $(\sim 340 \mathrm{~nm})$, suggesting that rGO may be able to cross the blood brain barrier and may be cleared through some as yet not well-understood mechanism.

For in vivo applications, further study is needed to characterize any potential brain region or cell-type specific effects. To date, most studies, such as detailed above, are performed in vitro. However, a few studies have characterized some of the effects of Gr/GO in vivo. Defteralí et al. (2016b) studied the effects of thermally reduced Gr on viability via stereotaxic injection into the mouse olfactory bulb. After 7 or 21 days, thermally reduced $\mathrm{Gr}$ had minimal effects on cell viability or number, and no significant increase in microglia number compared to injection only controls (Defteralí et al., 2016b). Tail vein injection of $>250 \mathrm{mg} / \mathrm{kg}$ dextran-modified $\mathrm{Gr}$ did not lead to brain toxicity after 30 days (Kanakia et al., 2014). However, this is not the route by which $\mathrm{Gr}$ would encounter the brain in most intended applications, thus requiring further toxicity study in vivo via direct Gr/GO injection to brain regions of interest. Although this has not been characterized for Gr/GObased materials, LDH activity has been shown to be differentially affected in a brain region-specific manner in multi-walled carbon nanotubes (MWNTs; Bussy et al., 2015). Thus, further study is warranted to determine if the toxicity is cell-type specific, as has been both suggested (Agarwal et al., 2010) and argued against (Ruiz et al., 2011).

\section{GRAPHENE AND NEURONS IN VITRO}

To date, even for in vitro systems, where there have been an array of studies using $\mathrm{Gr} / \mathrm{GO}$, questions remain as to toxicity. 


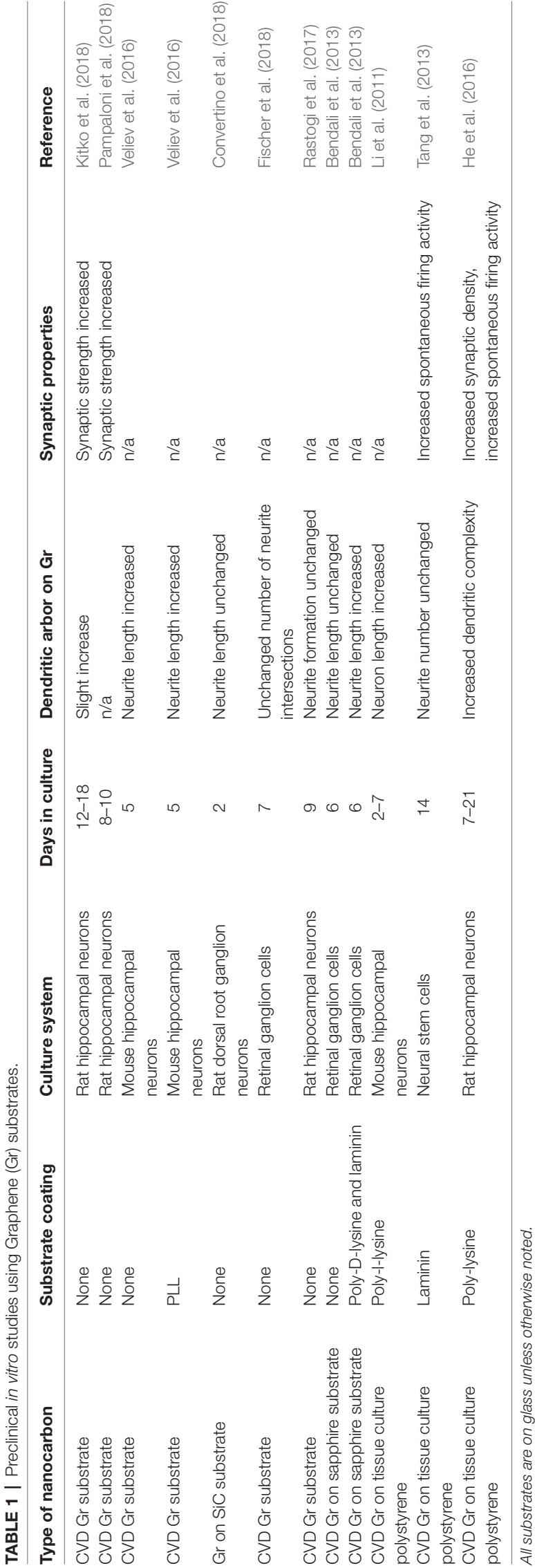

It is increasingly widely accepted that as a substrate for in vitro growth, $\mathrm{Gr}$ is a permissive surface both with and without the addition of extracellular matrix coatings (Table 1). Gr Flakes applied in culture have resulted in somewhat conflicting outcomes, but results generally support the idea that either or both high enough treatment dose or a long enough incubation time will result in cellular toxicity. Below these dosage or time thresholds, Gr flakes also have been studied for their ability to exert biological effects. Here, we move beyond toxicity to a discussion of hypotheses as to the causal underpinnings of biological changes reported on $\mathrm{Gr} / \mathrm{GO}$.

Most early studies using $\mathrm{Gr} / \mathrm{GO}$ substrates were conducted using a protein layer sandwiched between the substrate and neurons. For example, neural stem cells were grown on laminincoated tissue culture polystyrene and soaked in tissue culture media overnight (Tang et al., 2013). Chronic culture resulted in increases in: $\mathrm{Ca}^{2+}$ transient frequency, both spontaneous EPSC amplitude and frequency, and miniature EPSC frequency. These cellular changes occurred without altering overall stem cell morphology. Later versions of similar studies using neural stems cells did not observe changes in firing frequency, although were in overall agreement with increased cell signaling, here realized as increases in the percentage of cells firing action potentials during both proliferation and differentiation stages (Guo et al., 2016) and an increase in the density of neurites. Longer-term culture of stem cells on Gr also acts in a supportive manner by increasing overall cell count on Gr after 1 month (Park et al., 2011). Again, surface properties of $\mathrm{Gr} / \mathrm{GO}$ play an important role in stem cell proliferation and differentiation. For example, Defteralí et al. (2016a) used stem cells from adult mouse olfactory bulbs to test the effect of thermally reduced $\mathrm{Gr}$ and poly-vinylidene fluoride PVDF membranes loaded with multi-walled CNTs. Indeed, those nanomaterials significantly affect neurite branching and synapses during stem cell differentiation to neurons (Defteralí et al., 2016a,b). Similarly, changes during the differentiation of human induced pluripotent stem cells into multiple somatic cell lineages have been shown in graphene-treated samples (Yoo et al., 2014; Hu et al., 2015; Choi et al., 2016; Lee et al., 2016; RodriguezLosada et al., 2017; Wang et al., 2017; Nguyen et al., 2018; Sánchez-González et al., 2018; Saburi et al., 2019). The specific effects of $\mathrm{Gr} / \mathrm{GO}$ seem to be variable even within stem cell type, but overall results collectively suggest that Gr holds promise as a scaffold material for regenerative medicine and stem cell-based technologies.

Observations regarding the formation of synaptic connections, a fundamental unit of neuronal signaling, largely indicate that $\mathrm{Gr}$ is both permissive and to some extent may also enhance synaptic transmission. E18 cortical (Keshavan et al., 2018) or E18 hippocampal (Lorenzoni et al., 2013) neurons cultured on poly-d-lysine coated Gr "stripes" have been used to investigate synaptogenesis, with results indicating that functional synaptic connections are formed on Gr substrates covered with an adhesion coating. P0-P1 rat (He et al., 2016) or mouse hippocampal neurons grown on Gr coated with poly-lysine and pre-incubated in culture media demonstrated longer and more branched dendrites after 7 DIV and increased synapse number 
after 21 DIV (He et al., 2016), suggesting that the enhancements observed on Gr may be the result of some sort of conserved mechanism. This collection of studies has used pre-incubation in media overnight in addition to ECM coating, as it was elsewhere demonstrated to mitigate the cytotoxicity of $\mathrm{GO}$ (Hu et al., 2011). The observed decrease in cytotoxicity after overnight media incubation calls raises questions regarding the complex interactions between nanomaterial surfaces, the adhesion layer, and growth factors, lipids, etcetera, that are contained in fetal serums.

Increasing numbers of studies are interfacing directly to $\mathrm{Gr} / \mathrm{GO}$, omitting the intermediate protein-coating layer. This omits confounding factors both of additional surface charges due to the complex nature of such coatings and the physical gap created between the biological material of interest and the substrate. For example, differences in surface charge been shown to alter neurite outgrowth on GO (Tu et al., 2014), with positively charged surfaces overall exhibiting increased neurite outgrowth at 7 DIV. More broadly, coatings like polylysine are polycationic polymers, increasing cell attachment and outgrowth-but it is unclear whether there would be coupling between the coating and $\mathrm{Gr} / \mathrm{GO}$, masking direct biological effects. And recent studies have begun to systematically investigate the different biological effects observed even between different classes of ECM substrate (Fischer et al., 2018), suggesting that a single underlying mechanistic explanation of the biological effects of Gr must fully account for the composition of the substrate. The biological compatibility of Gr/GO substrates also does not appear to be cell-type specific, as retinal ganglion cells (Bendali et al., 2013; Fischer et al., 2018), cortical neurons (Rauti et al., 2016), hippocampal neurons (Veliev et al., 2016; Kitko et al., 2018; Pampaloni et al., 2018), and recently dorsal root ganglion neurons (Convertino et al., 2018) have all been cultured on bare Gr. However, neurite outgrowth and total number were reduced in comparison to a bare glass control for retinal ganglion cells (Bendali et al., 2013). Interestingly, this same study shows no significant enhancement on coated Gr compared to coated glass in these same properties, in contrast to much of the published literature that utilizes a protein coating layer; and later studies have made somewhat different observations, where neurons were not viable on bare glass controls but formed synaptic connections on uncoated Gr (Veliev et al., 2016). This also included comparisons to protein-coated Gr, where enhancements in neuronal surface area relative to bare Gr were observed over the duration of the study (up to $5 \mathrm{DIV}$ ). Although this and other studies have suggested that synaptic connections are formed on $\mathrm{Gr}$, later studies were left to determine synaptic function; and the effect of extracellular matrix addition on the ability to utilize the properties of $\mathrm{Gr}$ for neural recordings, for example, remains to be well-characterized.

The functional effects of $\mathrm{Gr}$ substrates have become an increasingly important area of study for neural interfacing applications. Multiple studies have now collectively suggested both that the frequency of neuronal firing is increased on bare Gr (Kitko et al., 2018; Pampaloni et al., 2018) and that synaptic strength is also increased (Rauti et al., 2016; Kitko et al., 2018), whereas high enough concentration treatment of neurons with
GO flakes reduces EPSC frequency (Rauti et al., 2016). However, there are somewhat conflicting explanations for the mechanisms underlying this synaptic enhancement. It has been hypothesized (Kitko et al., 2018) that chronic growth on Gr results in increased neuronal membrane cholesterol. This increase in cholesterol, possibly through the extraction of cholesterol from a serumcontaining media during the formation of a protein corona, is sufficient to explain the functional changes were observe on Gr. Specifically, Gr substrates result in an enlarged pool of synaptic vesicles and a higher vesicle release probability in neurons and potentiated $\mathrm{Ca}^{2+}$ release in $3 \mathrm{~T} 3$ cells. More recently, an alternative explanation has been proposed for the increased firing frequency on graphene (Pampaloni et al., 2018). Pampaloni et al. (2018) hypothesize that $\mathrm{K}^{+}$ions are depleted on bare graphene in the cleft between neurons and the substrate. This depletion of $\mathrm{K}^{+}$ions alters neuronal signaling, increasing EPSC frequency and altering adapting vs. tonic firing phenotypes. Computational models support this hypothesis, with the caveat that protein deposition during chronic culture is not included.

Although optical technologies hold great promise for pre-clinical application, to date, electrodes remain the most widely used and sought-after new technology for neural interfacing for clinical applications (Figure 2). Traditionally, electrodes are designed based upon a silicon manufacturing workflow, allowing for larger scale production that is currently available for Gr- and any carbon-based nanomaterials. Advances based upon this technology that may hold promise for a path to clinical applicability were recently demonstrated (Park et al., 2018). Gr electrodes with over $90 \%$ transmittance have been fabricated on parylene-C or polyethylene terephthalate (PET) substrates, permitting simultaneous optogenetic stimulation (Park et al., 2014; Liu et al., 2018), optical coherence tomography (Park et al., 2014), deep vasculature (Thunemann et al., 2018) or $\mathrm{Ca}^{2+}$ imaging (Park et al., 2018; Thunemann et al., 2018) in areas where the surface would normally be blocked by the opacity of traditional recording materials.

An ongoing area of research will be to improve the electronic properties of Gr to better improve recording quality while, at the same time, maintaining transparency and flexibility, important for in vivo imaging applications. Recent data have suggested that nanoparticle doping may be an approach to meet all of these requirements. For example, platinum nanoparticles electrodeposited onto CVD Gr overcome the quantum capacitance limits of $\mathrm{Gr}$ electrodes alone and enable improved signal quality ECoG and EEG signals (Lu et al., 2018). These signals can be simultaneously acquired with signals from genetically encoded indicators such as GCaMP at depths up to 250 microns using two-photon excitation.

\section{BEYOND THE MURINE MODEL: THE USE OF GRAPHENE IN OTHER MODEL SYSTEMS IN NEUROSCIENCE}

To date, most current understanding of the uses for and effects of $\mathrm{Gr}$ result from studies in murine models. Many of these studies are based on in vitro culture systems using neurons; future 


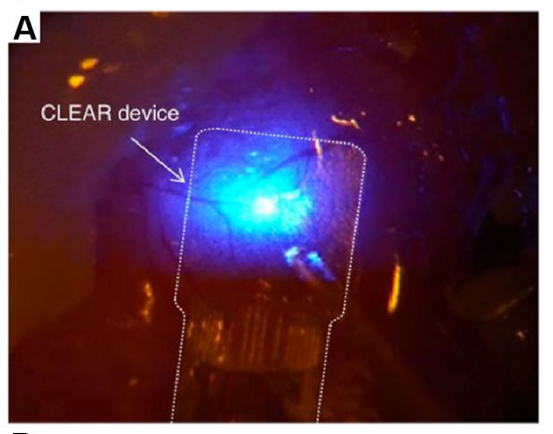

B

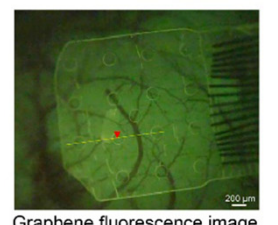

C
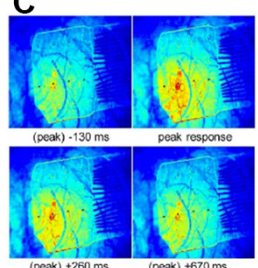

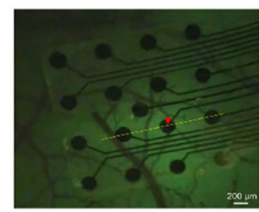

Platinum fluorescence image
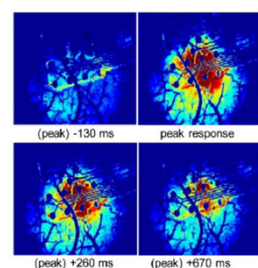
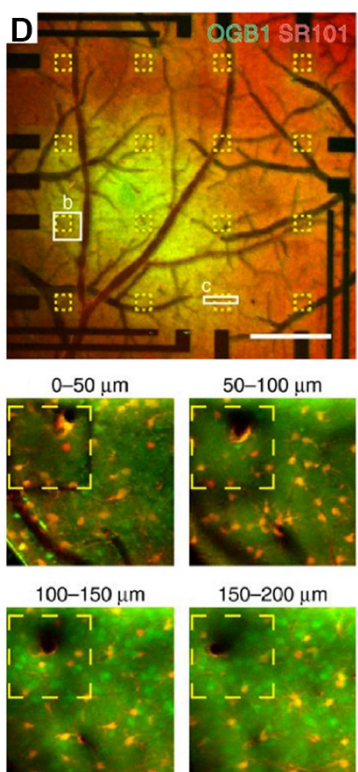

200-250 $\mu \mathrm{m}$

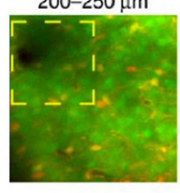

50-100 $\mu \mathrm{m}$

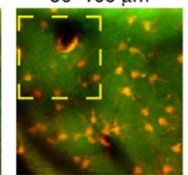

$150-200 \mu \mathrm{m}$

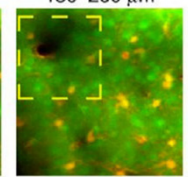

$250-300 \mu \mathrm{m}$

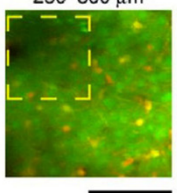

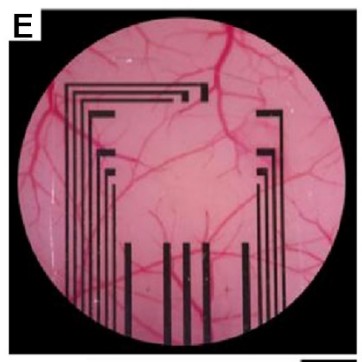
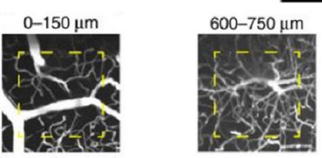

150-300 $\mu \mathrm{m}$

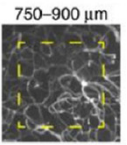

900-1050 $\mu \mathrm{m}$

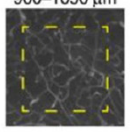

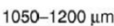

$450-600 \mu \mathrm{m}$
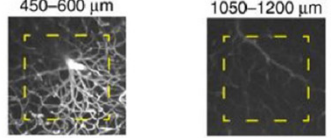

FIGURE 2 | Integration of transparent electrodes with complementary optical techniques in vivo. (A) Gr-based electrodes implanted on a mouse cerebral cortex. An optical fiber is also shown and used to deliver optogenetic stimulation. Modified with permission from Park et al. (2014). (B) Gr-based electrodes implanted on a mouse cerebral cortex (left) or traditional platinum electrodes Howe et al. (2013) and peak calcium responses from the same regions (C). Panels (B,C) are from Park et al. (2018). Copyright ACS Publication. (D) Two-photon calcium imaging to a depth of $300 \mu \mathrm{m}$ through Gr-based electrodes and intravascular imaging (E) to a depth of 1,200 $\mu \mathrm{m}$. Modified with permission from Thunemann et al. (2018).

directions should include mechanistic characterization of the biological effects of Gr/GO in vivo. The large array of transgenic modifications possible in mice and increasingly rats have enabled the study of ever more complex behaviors in a cell-type specific manner. These new techniques have resulted in a concurrent rise in the number of studies using other model systems, which have provided fundamental insights into the function of molecules, cells, circuits, and brain regions. Drosophila Melanogaster, for example, is a widely used system for the study of synapses and synaptic proteins (Keshishian et al., 1996). And historically, the sea slug Aplysia provided some of the earliest causal insight into the mechanisms of plasticity in the brain (Kandel, 2009). We here provide a general introduction to model systems where Gr/GO have been used to date.

The worm C. elegans is a soil nematode with 302 neurons in its nervous system, whose connectome, a map of all neural connections, has been characterized (Varshney et al., 2011; Jarrell et al., 2012), leading to ongoing efforts to develop causal rules governing structure and function relationships during behavior. It lacks a blood-brain barrier, enabling screening of molecules by delivery routes not available to traditional murine models. Although Gr-based device interfaces have yet to be demonstrated in C. elegans, Li et al. (2017) have demonstrated that chronic exposure to Gr elicits toxicity effects that are dosage-dependent, cell-type specific, and dependent upon the type of Gr. Nematodes typically have a lifetime of $\sim 2$ weeks, and underwent a 6-day chronic exposure that resulted in $\mathrm{Gr}$ being distributed throughout the digestive system. $100 \mathrm{mg} / \mathrm{L}$ Gr flakes did not significantly alter the overall survival rate after 6 days, but the same concentration of GO flakes was largely lethal. Interestingly, $>10 \mathrm{mg} / \mathrm{L}$ GO nanoparticles were shown to decrease expression levels of dat-1 and eat-4p, fluorescent genes encoding dopaminergic and glutamatergic neurons respectively, without significant downregulation of unc-47, which encodes GABAergic neurons. This is in contrast to the behavior of graphite nanoplatelets, where no acute in vivo toxicity was observed at concentrations up to $250 \mathrm{mg} / \mathrm{L}$ (Zanni et al., 2012). This could be due in part to differences in surface energy between GO and graphite nanoplatelets, for example, hydrophilic vs. hydrophobic wettability properties, but further investigation is warranted with more standardized concentrations between materials. As computational models and subsequent experiments have suggested that similar concentrations of Gr flakes should be destructive to the membrane, regardless of cell type (Luan et al., 2017), it would thus be helpful for future studies interested in 
assessing toxicity to take into account the concentrations used in previous studies for better cross-comparison.

Zebrafish represent another interesting possibility for demonstrating the utility of G/GO to the study of the brain. A developed zebrafish has $\sim 100,000$ neurons, fewer than any murine model, while still preserving many basic electrical and chemical signaling processes. This, combined with optical transparency, confers many advantages for single-cell resolution studies involving a whole population of neurons rather than the subsets that are optically accessible by even the most recent imaging approaches, for example, light field microscopy in freely moving rodents (Skocek et al., 2018). The availability of detailed genomic information and the relatively high degree of homology to the human genome ( $\sim 70 \%$; Howe et al., 2013) confer distinct advantages to their use as a model system. The relative ease of breeding and maintaining zebrafish and their short lifespan are also advantageous relative to rodents or non-human primates for reducing cost in larger scale toxicology screens (Fako and Furgeson, 2009). Although a concentration of $>120 \mathrm{mg} / \mathrm{L}$ singlewalled carbon nanotubes (SWNTs) was shown to delay hatching in zebrafish (Cheng et al., 2007), primary sensory neurons were not developmentally affected. Further studies using Gr/GO to assess toxicology in zebrafish are necessary for comparison to the effects observed using SWNTs.

The use of $\mathrm{Gr}$ has largely been limited in non-human primate models, due in part to lingering questions as to toxicity. However, organizations such as the European Graphene Flagship have issued calls for the production of Gr electrode arrays for recording in both non-human primates and humans. This will necessitate further study of the biological effects of $\mathrm{Gr}$ and Gr-based devices. As new strategies are developed to handle and integrate the vast and wide-ranging data streams becoming more prevalent in modern neuroscience, non-traditional model systems will continue to play a role in helping to elucidate the brain. Thus, future research on the compatibility of $\mathrm{Gr}$ with other model organisms will help to clarify the utility of $\mathrm{Gr}$ to these systems.

\section{REFERENCES}

Adamala, K. P., Martin-Alarcon, D. A., Guthrie-Honea, K. R., and Boyden, E. S. (2017). Engineering genetic circuit interactions within and between synthetic minimal cells. Nat. Chem. 9, 431-439. doi: 10.1038/nchem.2644

Agarwal, S., Zhou, X., Ye, F., He, Q., Chen, G. C. K., Soo, J., et al. (2010). Interfacing live cells with nanocarbon substrates. Langmuir 26, 2244-2247. doi: $10.1021 /$ la9048743

Akhavan, O. (2010). Graphene nanomesh by $\mathrm{ZnO}$ nanorod photocatalysts. ACS Nano 4, 4174-4180. doi: 10.1021/nn1007429

Akhavan, O., and Ghaderi, E. (2010). Toxicity of graphene and graphene oxide nanowalls against bacteria. ACS Nano 4, 5731-5736. doi: 10.1021/ nn $101390 x$

Akhavan, O., and Ghaderi, E. (2013). Graphene nanomesh promises extremely efficient in vivo photothermal therapy. Small 9, 3593-3601. doi: 10.1002/smll. 201203106

Bae, S., Kim, H., Lee, Y., Xu, X., Park, J.-S., Zheng, Y., et al. (2010). Roll-toroll production of 30 -inch graphene films for transparent electrodes. Nat. Nanotechnol. 5, 574-578. doi: 10.1038/nnano.2010.132

Bai, J., Zhong, X., Jiang, S., Huang, Y., and Duan, X. (2010). Graphene nanomesh. Nat. Nanotechnol. 5, 190-194. doi: 10.1038/nnano.2010.8

\section{PROSPECTS}

The ultimate utility of Gr will be determined in part by its ability to be used in conjunction with the large array and wide variety of optical, chemical, and electrical tools commonly utilized in modern neuroscience. The ability to combine fast optical control that is tuned via real-time device-based feedback is another promising direction (Kim et al., 2017).

Gr was quickly recognized as a "wonder material" after its isolation, including recognition as the 2010 Nobel Prize in physics. The number of publications referencing graphene has jumped to several thousand per year, a quick rise from several hundred only 10 years ago. Spurred on by several large initiatives, including the NIH's BRAIN and European Graphene Flagship, which represents the European Union's largest single research initiative, new applications for $\mathrm{Gr}$ to broad areas of brain research should continue to be developed at a rapid pace. Yet there are still challenges remaining, including addressing the widespread utility of many $\mathrm{Gr}$ applications. The promises of nanomedicine are extensive; what remains to be seen is the extent to which new applications for nanomaterials deliver on the great promise so often espoused.

\section{AUTHOR CONTRIBUTIONS}

KK and QZ conceived and wrote the article.

\section{FUNDING}

This work is funded by National Institutes of Health, NIH; Grant No. OD00876101 and NS094738 to QZ.

\section{ACKNOWLEDGMENTS}

We thank D. M. Bailey for comments and discussions. We thank all members of the Zhang laboratory for their support.

Bao, H., Pan, Y., Ping, Y., Sahoo, N. G., Wu, T., Li, L., et al. (2011). Chitosanfunctionalized graphene oxide as a nanocarrier for drug and gene delivery. Small 7, 1569-1578. doi: 10.1002/smll.201100191

Barroso-Bujans, F., Alegría, Á., and Colmenero, J. (2010). Kinetic study of the graphite oxide reduction: combined structural and gravimetric experiments under isothermal and nonisothermal conditions. J. Phys. Chem. C 114, 21645-21651. doi: 10.1021/jp108905j

Baskin, J. M., Prescher, J. A., Laughlin, S. T., Agard, N. J., Chang, P. V., Miller, I. A., et al. (2007). Copper-free click chemistry for dynamic in vivo imaging. Proc. Natl. Acad. Sci. U S A 104, 16793-16797. doi: 10.1073/pnas. 0707090104

Becerril, H. A., Mao, J., Liu, Z., Stoltenberg, R. M., Bao, Z., and Chen, Y. (2008). Evaluation of solution-processed reduced graphene oxide films as transparent conductors. ACS Nano 2, 463-470. doi: 10.1021/nn700375n

Belling, J. N., Jackman, J. A., Yorulmaz Avsar, S., Park, J. H., Wang, Y., Potroz, M. G., et al. (2016). Stealth immune properties of graphene oxide enabled by surface-bound complement factor H. ACS Nano 10, 10161-10172. doi: 10.1021/acsnano.6b05409

Bendali, A., Hess, L. H., Seifert, M., Forster, V., Stephan, A.-F., Garrido, J. A., et al. (2013). Purified neurons can survive on peptide-free graphene layers. $A d v$. Healthc. Mater. 2, 929-933. doi: 10.1002/adhm.201200347 
Bonaccorso, F., Lombardo, A., Hasan, T., Sun, Z., Colombo, L., and Ferrari, A. C. (2012). Production and processing of graphene and 2d crystals. Mater. Today 15, 564-589. doi: 10.1016/s1369-7021(13)70014-2

Bramini, M., Alberini, G., Colombo, E., Chiacchiaretta, M., DiFrancesco, M. L., Maya-Vetencourt, J. F., et al. (2018). Interfacing graphene-based materials with neural cells. Front. Syst. Neurosci. 12:12. doi: 10.3389/fnsys.2018.00012

Brodie, B. C. (1860). Sur le poids atomique du graphite. Ann. Chim. Phys. 59, $466-472$.

Bussy, C., Al-Jamal, K. T., Boczkowski, J., Lanone, S., Prato, M., Bianco, A., et al. (2015). Microglia determine brain region-specific neurotoxic responses to chemically functionalized carbon nanotubes. ACS Nano 9, 7815-7830. doi: 10.1021/acsnano.5b02358

Cao, L., Meziani, M. J., Sahu, S., and Sun, Y.-P. (2013). Photoluminescence properties of graphene versus other carbon nanomaterials. Acc. Chem. Res. 46, 171-180. doi: 10.1021/ar300128j

Castagnola, V., Zhao, W., Boselli, L., Lo Giudice, M. C., Meder, F., Polo, E., et al. (2018). Biological recognition of graphene nanoflakes. Nat. Commun. 9:1577. doi: 10.1038/s41467-018-04009-x

Cedervall, T., Lynch, I., Lindman, S., Berggård, T., Thulin, E., Nilsson, H., et al. (2007). Understanding the nanoparticle-protein corona using methods to quantify exchange rates and affinities of proteins for nanoparticles. Proc. Natl. Acad. Sci. U S A 104, 2050-2055. doi: 10.1073/pnas.0608582104

Cellot, G., Lagonegro, P., Tarabella, G., Scaini, D., Fabbri, F., Iannotta, S., et al. (2016). PEDOT:PSS interfaces support the development of neuronal synaptic networks with reduced neuroglia response in vitro. Front. Neurosci. 9:521. doi: 10.3389/fnins.2015.00521

Chaudhary, U., Birbaumer, N., and Ramos-Murguialday, A. (2016). Braincomputer interfaces for communication and rehabilitation. Nat. Rev. Neurol. 12, 513-525. doi: 10.1038/nrneurol.2016.113

Chen, F., Tillberg, P. W., and Boyden, E. S. (2015). Expansion microscopy. Science 347, 543-548. doi: 10.1126/science. 1260088

Chen, S., Xiong, C., Liu, H., Wan, Q., Hou, J., He, Q., et al. (2015). Mass spectrometry imaging reveals the sub-organ distribution of carbon nanomaterials. Nat. Nanotechnol. 10, 176-182. doi: 10.1038/nnano. 2014.282

Cheng, J., Flahaut, E., and Cheng, S. H. (2007). Effect of carbon nanotubes on developing zebrafish (Danio Rerio) embryos. Environ. Toxicol. Chem. 26, 708-716. doi: 10.1897/06-272r.1

Choi, M., Kim, K.-G., Heo, J., Jeong, H., Kim, S. Y., and Hong, J. (2015). Multilayered graphene nano-film for controlled protein delivery by desired electro-stimuli. Sci. Rep. 5:17631. doi: 10.1038/srep17631

Choi, H. Y., Lee, T. J., Yang, G. M., Oh, J., Won, J., Han, J., et al. (2016). Efficient mRNA delivery with graphene oxide-polyethylenimine for generation of footprint-free human induced pluripotent stem cells. J. Control. Release 235, 222-235. doi: 10.1016/j.jconrel.2016.06.007

Chong, Y., Ge, C., Yang, Z., Garate, J. A., Gu, Z., Weber, J. K., et al. (2015). Reduced cytotoxicity of graphene nanosheets mediated by blood-protein coating. ACS Nano 9, 5713-5724. doi: 10.1021/nn5066606

Chung, K., Wallace, J., Kim, S.-Y., Kalyanasundaram, S., Andalman, A. S., Davidson, T. J., et al. (2013). Structural and molecular interrogation of intact biological systems. Nature 497, 332-337. doi: 10.1038/nature12107

Convertino, D., Luin, S., Marchetti, L., and Coletti, C. (2018). Peripheral neuron survival and outgrowth on graphene. Front. Neurosci. 12:1. doi: 10.3389/fnins. 2018.00001

Defteralí, Ç., Verdejo, R., Majeed, S., Boschetti-de-Fierro, A., MéndezGómez, H. R., Díaz-Guerra, E., et al. (2016a). In vitro evaluation of biocompatibility of uncoated thermally reduced graphene and carbon nanotube-loaded PVDF membranes with adult neural stem cell-derived neurons and glia. Front. Bioeng. Biotechnol. 4:94. doi: 10.3389/fbioe.2016.00094

Defteralí, Ç., Verdejo, R., Peponi, L., Martín, E. D., Martínez-Murillo, R., LópezManchado, M. Á., et al. (2016b). Thermally reduced graphene is a permissive material for neurons and astrocytes and de novo neurogenesis in the adult olfactory bulb in vivo. Biomaterials 82, 84-93. doi: 10.1016/j.biomaterials.2015. 12.010

Ding, X., Liu, H., and Fan, Y. (2015). Graphene-based materials in regenerative medicine. Adv. Healthc. Mater. 4, 1451-1468. doi: 10.1002/adhm.201500203

Domínguez-Bajo, A., González-Mayorga, A., López-Dolado, E., and Serrano, M. C. (2017). Graphene-derived materials interfacing the spinal cord: outstanding in vitro and in vivo findings. Front. Syst. Neurosci. 11:71. doi: $10.3389 /$ fnsys.2017.00071

Dong, H., Jin, M., Liu, Z., Xiong, H., Qiu, X., Zhang, W., et al. (2016). In vitro and in vivo brain-targeting chemo-photothermal therapy using graphene oxide conjugated with transferrin for Gliomas. Lasers Med. Sci. 31, 1123-1131. doi: 10.1007/s10103-016-1955-2

Dong, H., Li, Y., Yu, J., Song, Y., Cai, X., Liu, J., et al. (2013). A versatile multicomponent assembly via $\beta$-cyclodextrin host-guest chemistry on graphene for biomedical applications. Small 9, 446-456. doi: 10.1002/smll. 201201003

Donoghue, J. P., Nurmikko, A., Black, M., and Hochberg, L. R. (2007). Assistive technology and robotic control using motor cortex ensemble-based neural interface systems in humans with tetraplegia. J. Physiol. 579, 603-611. doi: 10.1113/jphysiol.2006.127209

Du, X., Wu, L., Cheng, J., Huang, S., Cai, Q., Jin, Q., et al. (2015). Graphene microelectrode arrays for neural activity detection. J. Biol. Phys. 41, 339-347. doi: 10.1007/s10867-015-9382-3

Duan, G., Kang, S.-G., Tian, X., Garate, J. A., Zhao, L., Ge, C., et al. (2015). Protein corona mitigates the cytotoxicity of graphene oxide by reducing its physical interaction with cell membrane. Nanoscale 7, 15214-15224. doi: $10.1039 / \mathrm{c} 5 \mathrm{nr} 01839 \mathrm{k}$

Eda, G., Fanchini, G., and Chhowalla, M. (2008). Large-area ultrathin films of reduced graphene oxide as a transparent and flexible electronic material. Nat. Nanotechnol. 3, 270-274. doi: 10.1038/nnano.2008.83

Fako, V. E., and Furgeson, D. Y. (2009). Zebrafish as a correlative and predictive model for assessing biomaterial nanotoxicity. Adv. Drug Deliv. Rev. 61, 478-486. doi: 10.1016/j.addr.2009.03.008

Fang, M., Long, J., Zhao, W., Wang, L., and Chen, G. (2010). pH-responsive chitosan-mediated graphene dispersions. Langmuir 26, 16771-16774. doi: $10.1021 /$ la102703b

Faria, M., Björnmalm, M., Thurecht, K. J., Kent, S. J., Parton, R. G., Kavallaris, M., et al. (2018). Minimum information reporting in bio-nano experimental literature. Nat. Nanotechnol. 13, 777-785. doi: 10.1038/s41565-0180246-4

Fattahi, P., Yang, G., Kim, G., and Abidian, M. R. (2014). A review of organic and inorganic biomaterials for neural interfaces. Adv. Mater. 26, 1846-1885. doi: 10.1002/adma.201304496

Feng, L., Zhang, S., and Liu, Z. (2011). Graphene based gene transfection. Nanoscale 3, 1252-1257. doi: 10.1039/c0nr00680g

Fiori, G., Bonaccorso, F., Iannaccone, G., Palacios, T., Neumaier, D., Seabaugh, A., et al. (2014). Electronics based on two-dimensional materials. Nat. Nanotechnol. 9, 768-779. doi: 10.1038/nnano.2014.207

Fischer, R. A., Zhang, Y., Risner, M. L., Li, D., Xu, Y., and Sappington, R. M. (2018). Impact of graphene on the efficacy of neuron culture substrates. Adv. Healthc. Mater. 7:e1701290. doi: 10.1002/adhm.201701290

Frost, R., Jönsson, G. E., Chakarov, D., Svedhem, S., and Kasemo, B. (2012). Graphene oxide and lipid membranes: interactions and nanocomposite structures. Nano Lett. 12, 3356-3362. doi: 10.1021/nl203107k

Geim, A. K., and Novoselov, K. S. (2007). The rise of graphene. Nat. Mater. 6, 183-191. doi: 10.1038/nmat1849

Gómez-Navarro, C., Weitz, R. T., Bittner, A. M., Scolari, M., Mews, A., Burghard, M., et al. (2007). Electronic transport properties of individual chemically reduced graphene oxide sheets. Nano Lett. 7, 3499-3503. doi: 10.1021/nl072090c

Gu, Y., Guo, Y., Wang, C., Xu, J., Wu, J., Kirk, T. B., et al. (2017). A polyamidoamne dendrimer functionalized graphene oxide for DOX and MMP-9 shRNA plasmid co-delivery. Mater. Sci. Eng. C Mater. Biol. Appl. 70, 572-585. doi: 10.1016/j.msec.2016.09.035

Guo, R., Mao, J., and Yan, L.-T. (2013). Computer simulation of cell entry of graphene nanosheet. Biomaterials 34, 4296-4301. doi: 10.1016/j.biomaterials. 2013.02.047

Guo, R., Zhang, S., Xiao, M., Qian, F., He, Z., Li, D., et al. (2016). Accelerating bioelectric functional development of neural stem cells by graphene coupling: implications for neural interfacing with conductive materials. Biomaterials 106 , 193-204. doi: 10.1016/j.biomaterials.2016.08.019

Han, T.-H., Kwon, S.-J., Li, N., Seo, H.-K., Xu, W., Kim, K. S., et al. (2016). Versatile p-type chemical doping to achieve ideal flexible graphene electrodes. Angew. Chem. Int. Ed. Engl. 55, 6197-6201. doi: 10.1002/anie.201600414 
He, Z., Zhang, S., Song, Q., Li, W., Liu, D., Li, H., et al. (2016). The structural development of primary cultured hippocampal neurons on a graphene substrate. Colloids Surf. B Biointerfaces 146, 442-451. doi: 10.1016/j.colsurfb. 2016.06.045

Hernandez, Y., Nicolosi, V., Lotya, M., Blighe, F. M., Sun, Z., De, S., et al. (2008). High-yield production of graphene by liquid-phase exfoliation of graphite. Nat. Nanotechnol. 3, 563-568. doi: 10.1038/nnano.2008.215

Hochberg, L. R., Serruya, M. D., Friehs, G. M., Mukand, J. A., Saleh, M., Caplan, A. H., et al. (2006). Neuronal ensemble control of prosthetic devices by a human with tetraplegia. Nature 442, 164-471. doi: 10.1038/nature04970

Hong, J., Shah, N. J., Drake, A. C., DeMuth, P. C., Lee, J. B., Chen, J., et al. (2012). Graphene multilayers as gates for multi-week sequential release of proteins from surfaces. ACS Nano 6, 81-88. doi: 10.1021/nn202607r

Howe, K., Clark, M. D., Torroja, C. F., Torrance, J., Berthelot, C., Muffato, M., et al. (2013). The zebrafish reference genome sequence and its relationship to the human genome. Nature 496, 498-503. doi: 10.1038/nature12111

Hu, W., Peng, C., Lv, M., Li, X., Zhang, Y., Chen, N., et al. (2011). Protein coronamediated mitigation of cytotoxicity of graphene oxide. ACS Nano 5, 3693-3700. doi: $10.1021 / \mathrm{nn} 200021 \mathrm{j}$

Hu, S., Zeng, Y., Yang, S., Qin, H., Cai, H., and Wang, J. (2015). Application of graphene based nanotechnology in stem cells research. J. Nanosci. Nanotechnol. 15, 6327-6341. doi: 10.1166/jnn.2015.10844

Hummers, W. S., and Offeman, R. E. (1958). Preparation of graphitic oxide. J. Am. Chem. Soc. 80:1339. doi: 10.1021/ja01539a017

Jan, E., Hendricks, J. L., Husaini, V., Richardson-Burns, S. M., Sereno, A., Martin, D. C., et al. (2009). Layered carbon nanotube-polyelectrolyte electrodes outperform traditional neural interface materials. Nano Lett. 9, 4012-4018. doi: $10.1021 / \mathrm{nl} 902187 \mathrm{z}$

Jarrell, T. A., Wang, Y., Bloniarz, A. E., Brittin, C. A., Xu, M., Thomson, J. N., et al. (2012). The connectome of a decision-making neural network. Science 337, 437-444. doi: 10.1126/science.1221762

Jung, I., Field, D. A., Clark, N. J., Zhu, Y., Yang, D., Piner, R. D., et al. (2009). Reduction kinetics of graphene oxide determined by electrical transport measurements and temperature programmed desorption. J. Phys. Chem. C 113, 18480-18486. doi: 10.1021/jp904396j

Kaminska, I., Das, M. R., Coffinier, Y., Niedziolka-Jonsson, J., Sobczak, J., Woisel, P., et al. (2012). Reduction and functionalization of graphene oxide sheets using biomimetic dopamine derivatives in one step. ACS Appl. Mater. Interfaces 4, 1016-1020. doi: 10.1021/am201664n

Kanakia, S., Toussaint, J. D., Mullick Chowdhury, S., Tembulkar, T., Lee, S., Jiang, Y.-P., et al. (2014). Dose ranging, expanded acute toxicity and safety pharmacology studies for intravenously administered functionalized graphene nanoparticle formulations. Biomaterials 35, 7022-7031. doi: 10.1016/j. biomaterials.2014.04.066

Kandel, E. R. (2009). The biology of memory: a forty-year perspective. J. Neurosci. 29, 12748-12756. doi: 10.1523/jneurosci.3958-09.2009

Keshavan, S., Naskar, S., Diaspro, A., Cancedda, L., and Dante, S. (2018). Developmental refinement of synaptic transmission on micropatterned single layer graphene. Acta Biomater. 65, 363-375. doi: 10.1016/j.actbio.2017. 11.005

Keshishian, H., Broadie, K., Chiba, A., and Bate, M. (1996). The drosophila neuromuscular junction: a model system for studying synaptic development and function. Ann. Rev. Neurosci. 19, 545-575. doi: 10.1146/annurev.neuro.19. 1.545

Kim, C. K., Adhikari, A., and Deisseroth, K. (2017). Integration of optogenetics with complementary methodologies in systems neuroscience. Nat. Rev. Neurosci. 18, 222-235. doi: 10.1038/nrn.2017.15

Kim, H., Lee, D., Kim, J., Kim, T.-I., and Kim, W. J. (2013). Photothermally triggered cytosolic drug delivery via endosome disruption using a functionalized reduced graphene oxide. ACS Nano 7, 6735-6746. doi: $10.1021 / \mathrm{nn} 403096 \mathrm{~s}$

Kitko, K. E., Hong, T., Lazarenko, R. M., Ying, D., Xu, Y.-Q., and Zhang, Q. (2018). Membrane cholesterol mediates the cellular effects of monolayer graphene substrates. Nat. Commun. 9:796. doi: 10.1038/s41467-01803185-0

Kostarelos, K., Bianco, A., and Prato, M. (2009). Promises, facts and challenges for carbon nanotubes in imaging and therapeutics. Nat. Nanotechnol. 4, 627-633. doi: $10.1038 /$ nnano.2009.241
Kurapati, R., and Raichur, A. M. (2012). Graphene oxide based multilayer capsules with unique permeability properties: facile encapsulation of multiple drugs. Chem. Commun. 48, 6013-6015. doi: 10.1039/c2cc32248j

Kuzum, D., Takano, H., Shim, E., Reed, J. C., Juul, H., Richardson, A. G., et al. (2014). Transparent and flexible low noise graphene electrodes for simultaneous electrophysiology and neuroimaging. Nat. Commun. 5:5259. doi: $10.1038 /$ ncomms6259

Lee, H., Nam, D., Choi, J. K., Arauzo-Bravo, M. J., Kwon, S. Y., Zaehres, H., et al. (2016). Establishment of feeder-free culture system for human induced pluripotent stem cell on DAS nanocrystalline graphene. Sci. Rep. 6:20708 doi: 10.1038/srep20708

Lee, C., Wei, X., Kysar, J. W., and Hone, J. (2008). Measurement of the elastic properties and intrinsic strength of monolayer graphene. Science 321, 385-388. doi: 10.1126/science.1157996

Lei, H., Zhou, X., Wu, H., Song, Y., Hu, J., Guo, S., et al. (2014). Morphology change and detachment of lipid bilayers from the mica substrate driven by graphene oxide sheets. Langmuir 30, 4678-4683. doi: 10.1021/la500788z

Li, X., Cai, W., An, J., Kim, S., Nah, J., Yang, D., et al. (2009a). Large-area synthesis of high-quality and uniform graphene films on copper foils. Science 324, 1312-1314. doi: 10.1126/science.1171245

Li, X., Wang, H., Robinson, J. T., Sanchez, H., Diankov, G., and Dai, H. (2009b). Simultaneous nitrogen doping and reduction of graphene oxide. J. Am. Chem. Soc. 131, 15939-15944. doi: 10.1021/ja907098f

Li, Y., Feng, L., Shi, X., Wang, X., Yang, Y., Yang, K., et al. (2014). Surface coatingdependent cytotoxicity and degradation of graphene derivatives: towards the design of non-toxic, degradable nano-graphene. Small 10, 1544-1554. doi: 10.1002/smll.201303234

Li, D., Müller, M. B., Gilje, S., Kaner, R. B., and Wallace, G. G. (2008). Processable aqueous dispersions of graphene nanosheets. Nat. Nanotechnol. 3, 101-105. doi: 10.1038/nnano.2007.451

Li, S., Stein, A. J., Kruger, A., and Leblanc, R. M. (2013). Head groups of lipids govern the interaction and orientation between graphene oxide and lipids. J. Phys. Chem. C 117, 16150-16158. doi: 10.1021/jp405991q

Li, P., Xu, T., Wu, S., Lei, L., and He, D. (2017). Chronic exposure to graphene-based nanomaterials induces behavioral deficits and neural damage in Caenorhabditis elegans. J. Appl. Toxicol. 37, 1140-1150. doi: 10.1002/ jat.3468

Li, M., Yang, X., Ren, J., Qu, K., and Qu, X. (2012). Using graphene oxide high near-infrared absorbance for photothermal treatment of Alzheimer's disease. Adv. Mater. 24, 1722-1728. doi: 10.1002/adma.201104864

Li, Y., Yuan, H., von dem Bussche, A., Creighton, M., Hurt, R. H., Kane, A. B., et al. (2013). Graphene microsheets enter cells through spontaneous membrane penetration at edge asperities and corner sites. Proc. Natl. Acad. Sci. U S A 110, 12295-12300. doi: 10.1073/pnas.1222276110

Li, N., Zhang, X., Song, Q., Su, R., Zhang, Q., Kong, T., et al. (2011). The promotion of neurite sprouting and outgrowth of mouse hippocampal cells in culture by grapheme substrates. Biomaterials 32, 9374-9382. doi: 10.1016/j.biomaterials.2011.08.065

Little, S., Pogosyan, A., Neal, S., Zavala, B., Zrinzo, L., Hariz, M., et al. (2013). Adaptive deep brain stimulation in advanced Parkinson disease. Ann. Neurol. 74, 449-457. doi: 10.1002/ana.23951

Liu, N., Chortos, A., Lei, T., Jin, L., Kim, T. R., Bae, W.-G., et al. (2017). Ultratransparent and stretchable graphene electrodes. Sci. Adv. 3:e1700159. doi: 10.1126/sciadv.1700159

Liu, X., Lu, Y., Iseri, E., Shi, Y., and Kuzum, D. (2018). A compact closed-loop optogenetics system based on artifact-free transparent graphene electrodes. Front. Neurosci. 12:132. doi: 10.3389/fnins.2018.00132

Liu, X., Miller, A. L. II., Park, S., Waletzki, B. E., Zhou, Z., Terzic, A., et al. (2017). Functionalized carbon nanotube and graphene oxide embedded electrically conductive hydrogel synergistically stimulates nerve cell differentiation. ACS Appl. Mater. Interfaces 9, 14677-14690. doi: 10.1021/acsami. $7 \mathrm{~b} 02072$

Liu, Z., Robinson, J. T., Tabakman, S. M., Yang, K., and Dai, H. (2011). Carbon materials for drug delivery and cancer therapy. Mater. Today 14, 316-323. doi: 10.1016/S1369-7021(11)70161-4

Liu, Z., Robinson, J. T., Sun, X., and Dai, H. (2008). PEGylated nanographene oxide for delivery of water-insoluble cancer drugs. J. Am. Chem. Soc. 130, 10876-10877. doi: 10.1021/ja803688x 
Liu, G., Shen, H., Mao, J., Zhang, L., Jiang, Z., Sun, T., et al. (2013). Transferrin modified graphene oxide for glioma-targeted drug delivery: in vitro and in vivo evaluations. ACS Appl. Mater. Interfaces 5, 6909-6914. doi: 10.1021/ am402128s

Liu, C.-C., Zhao, J.-J., Zhang, R., Li, H., Chen, B., L.-Zhang, L., et al. (2017). Multifunctionalization of graphene and graphene oxide for controlled release and targeted delivery of anticancer drugs. Am. J. Transl. Res. 9, 5197-5219. doi: 10.1126/sciadv.1700159

Lorenzoni, M., Brandi, F., Dante, S., Giugni, A., and Torre, B. (2013). Simple and effective graphene laser processing for neuron patterning application. Sci. Rep. 3:1954. doi: 10.1038/srep01954

Lotya, M., Hernandez, Y., King, P. J., Smith, R. J., Nicolosi, V., Karlsson, L. S., et al. (2009). Liquid phase production of graphene by exfoliation of graphite in surfactant/water solutions. J. Am. Chem. Soc. 131, 3611-3620. doi: 10.1021/ja807449u

Lu, Y., Liu, X., Hattori, R., Ren, C., Zhang, X., Komiyama, T., et al. (2018). Ultralow impedance graphene microelectrodes with high optical transparency for simultaneous deep two-photon imaging in transgenic mice. Adv. Funct. Mater. 28:1800002. doi: 10.1002/adfm.201800002

Lu, C.-H., Zhu, C.-L., Li, J., Liu, J.-J., Chen, X., and Yang, H.-H. (2010). Using graphene to protect DNA from cleavage during cellular delivery. Chem. Commun. 46, 3116-3118. doi: 10.1039/b926893f

Luan, B., Zhou, S., Wang, D., and Zhou, R. (2017). Detecting interactions between nanomaterials and cell membranes by synthetic nanopores. ACS Nano 11, 12615-12623. doi: 10.1021/acsnano.7b07005

Luo, Z., Vora, P. M., Mele, E. J., Johnson, A. T. C., and Kikkawa, J. M. (2009). Photoluminescence and band gap modulation in graphene oxide. Appl. Phys. Lett. 94:111909. doi: 10.1063/1.3098358

Luo, X., Weaver, C. L., Zhou, D. D., Greenberg, R., and Cui, X. T. (2011). Highly stable carbon nanotube doped poly(3,4-ethylenedioxythiophene) for chronic neural stimulation. Biomaterials 32, 5551-5557. doi: 10.1016/j.biomaterials. 2011.04.051

Markovic, Z. M., Harhaji-Trajkovic, L. M., Todorovic-Markovic, B. M., Kepic, D. P., Arsikin, K. M., Jovanovic, S. P., et al. (2011). in vitro comparison of the photothermal anticancer activity of graphene nanoparticles and carbon nanotubes. Biomaterials 32, 1121-1129. doi: 10.1016/j.biomaterials.2010. 10.030

Matteini, P., Tatini, F., Cavigli, L., Ottaviano, S., Ghini, G., and Pini, R. (2014). Graphene as a photothermal switch for controlled drug release. Nanoscale 6, 7947-7953. doi: 10.1039/c4nr01622j

Mendonça, M. C. P., Soares, E. S., de Jesus, M. B., Ceragioli, H. J., Ferreira, M. S., Catharino, R. R., et al. (2015). Reduced graphene oxide induces transient blood-brain barrier opening: an in vivo study. J. Nanobiotechnol. 13:78. doi: 10.1186/s12951-015-0143-Z

Moon, I. K., Lee, J., Ruoff, R. S., and Lee, H. (2010). Reduced graphene oxide by chemical graphitization. Nat. Commun. 1:73. doi: 10.1038/ncomms1067

Nel, A. E., Mädler, L., Velegol, D., Xia, T., Hoek, E. M. V., Somasundaran, P., et al. (2009). Understanding biophysicochemical interactions at the nano-bio interface. Nat. Mater. 8, 543-557. doi: 10.1038/nmat 2442

Nguyen, A. T., Mattiassi, S., Loeblein, M., Chin, E., Ma, D., Coquet, P., et al. (2018). Human Rett-derived neuronal progenitor cells in 3D graphene scaffold as an in vitro platform to study the effect of electrical stimulation on neuronal differentiation. Biomed. Mater. 13:034111. doi: 10.1088/1748-605x/aaaf2b

Nicholl, R. J. T., Conley, H. J., Lavrik, N. V., Vlassiouk, I., Puzyrev, Y. S., Sreenivas, V. P., et al. (2015). The effect of intrinsic crumpling on the mechanics of free-standing graphene. Nat. Commun. 6:8789. doi: 10.1038/ncomms9789

Novoselov, K. S., Geim, A. K., Morozov, S. V., Jiang, D., Zhang, Y., Dubonos, S. V., et al. (2004). Electric field effect in atomically thin carbon Films. Science 306, 666-669. doi: 10.1126/science.1102896

Novoselov, K. S., Jiang, D., Schedin, F., Booth, T. J., Khotkevich, V. V., Morozov, S. V., et al. (2005). Two-dimensional atomic crystals. Proc. Natl. Acad. Sci. U S A 102, 10451-10453. doi: 10.1073/pnas.0502848102

Pampaloni, N. P., Lottner, M., Giugliano, M., Matruglio, A., D’Amico, F., Prato, M., et al. (2018). Single-layer graphene modulates neuronal communication and augments membrane ion currents. Nat. Nanotechnol. 13, 755-764. doi: 10.1038/s41565-018-0163-6

Pang, J., Mendes, R. G., Wrobel, P. S., Wlodarski, M. D., Ta, H. Q., Zhao, L., et al. (2017). Self-terminating confinement approach for large-area uniform monolayer graphene directly over $\mathrm{Si} / \mathrm{SiO}_{x}$ by chemical vapor deposition. ACS Nano 11, 1946-1956. doi: 10.1021/acsnano.6b08069

Park, D.-W., Brodnick, S. K., Ness, J. P., Atry, F., Krugner-Higby, L., Sandberg, A., et al. (2016). Fabrication and utility of a transparent graphene neural electrode array for electrophysiology, in vivo imaging, and optogenetics. Nat. Protoc. 11, 2201-2222. doi: 10.1038/nprot.2016.127

Park, D.-W., Ness, J. P., Brodnick, S. K., Esquibel, C., Novello, J., Atry, F., et al. (2018). Electrical neural stimulation and simultaneous in vivo monitoring with transparent graphene electrode arrays implanted in GCaMP6f mice. ACS Nano 12, 148-157. doi: 10.1021/acsnano.7b04321

Park, S. Y., Park, J., Sim, S. H., Sung, M. G., Kim, K. S., Hong, B. H., et al. (2011). Enhanced differentiation of human neural stem cells into neurons on graphene. Adv. Mater. 23, H263-H267. doi: 10.1002/adma.201101503

Park, S., and Ruoff, R. S. (2009). Chemical methods for the production of graphenes. Nat. Nanotechnol. 4, 217-224. doi: 10.1038/nnano.2009.58

Park, D.-W., Schendel, A. A., Mikael, S., Brodnick, S. K., Richner, T. J., Ness, J. P., et al. (2014). Graphene-based carbon-layered electrode array technology for neural imaging and optogenetic applications. Nat. Commun. 5:5258. doi: $10.1038 /$ ncomms6258

Paton, K. R., Varrla, E., Backes, C., Smith, R. J., Khan, U., O'Neill, A., et al. (2014). Scalable production of large quantities of defect-free few-layer graphene by shear exfoliation in liquids. Nat. Mater. 13, 624-630. doi: 10.1038/ nmat3944

Polikov, V. S., Tresco, P. A., and Reichert, W. M. (2005). Response of brain tissue to chronically implanted neural electrodes. J. Neurosci. Methods 148, 1-18. doi: 10.1016/j.jneumeth.2005.08.015

Qian, J., Wang, D., Cai, F.-H., Xi, W., Peng, L., Zhu, Z.-F., et al. (2012). Observation of multiphoton-induced fluorescence from graphene oxide nanoparticles and applications in in vivo functional bioimaging. Angew. Chem. Int. Ed. Engl. 51, 10570-10575. doi: 10.1002/anie.201206107

Quintana, M., Montellano, A., del Rio Castillo, A. E., Tendeloo, G. V., Bittencourt, C., and Prato, M. (2011). Selective organic functionalization of graphene bulk or graphene edges. Chem. Commun. 47, 9330-9332. doi: $10.1039 / \mathrm{clcc} 13254 \mathrm{~g}$

Radic, S., Geitner, N. K., Podila, R., Käkinen, A., Chen, P., Ke, P. C., et al. (2013). Competitive binding of natural amphiphiles with graphene derivatives. Sci. Rep. 3:2273. doi: 10.1038/srep02273

Ramos-Murguialday, A., Broetz, D., Rea, M., Läer, L., Yilmaz, Ö., Brasil, F. L., et al. (2013). Brain-machine interface in chronic stroke rehabilitation: a controlled study. Ann. Neurol. 74, 100-108. doi: 10.1002/ana.23879

Rastogi, S. K., Raghavan, G., Yang, G., and Cohen-Karni, T. (2017). Effect of graphene on nonneuronal and neuronal cell viability and stress. Nano Lett. 17, 3297-3301. doi: 10.1021/acs.nanolett.7b01215

Rauti, R., Lozano, N., León, V., Scaini, D., Musto, M., Rago, I., et al. (2016). Graphene oxide nanosheets reshape synaptic function in cultured brain networks. ACS Nano 10, 4459-4471. doi: 10.1021/acsnano.6b00130

Reina, A., Jia, X., Ho, J., Nezich, D., Son, H., Bulovic, V., et al. (2009). Large area, few-layer graphene films on arbitrary substrates by chemical vapor deposition. Nano Lett. 9, 30-35. doi: 10.1021/nl801827v

Richardson-Burns, S. M., Hendricks, J. L., Foster, B., Povlich, L. K., Kim, D.-H., and Martin, D. C. (2007). Polymerization of the conducting polymer poly $(3,4-$ ethylenedioxythiophene) (PEDOT) around living neural cells. Biomaterials 28, 1539-1552. doi: 10.1016/j.biomaterials.2006.11.026

Rivnay, J., Wang, H., Fenno, L., Deisseroth, K., and Malliaras, G. G. (2017). Next-generation probes, particles, and proteins for neural interfacing. Sci. Adv. 3:e1601649. doi: 10.1126/sciadv.1601649

Robinson, J. T., Tabakman, S. M., Liang, Y., Wang, H., Casalongue, H. S., Vinh, D., et al. (2011). Ultrasmall reduced graphene oxide with high near-infrared absorbance for photothermal therapy. J. Am. Chem. Soc. 133, 6825-6831. doi: 10.1021/ja2010175

Robinson, D. L., Venton, B. J., Heien, M. L. A. V., and Wightman, R. M. (2003). Detecting subsecond dopamine release with fast-scan cyclic voltammetry in vivo. Clin. Chem. 49, 1763-1773. doi: 10.1373/49.10.1763

Rodriguez-Losada, N., Romero, P., Estivill-Torrús, G., Guzmán de Villoria, R., and Aguirre, J. A. (2017). Cell survival and differentiation with nanocrystalline glass-like carbon using substantia nigra dopaminergic cells derived from transgenic mouse embryos. PLoS One 12:e0173978. doi: 10.1371/journal.pone. 0173978 
Romero-Aburto, R., Narayanan, T. N., Nagaoka, Y., Hasumura, T., Mitcham, T. M., Fukuda, T., et al. (2013). Fluorinated graphene oxide; a new multimodal material for biological applications. Adv. Mater. 25, 5632-5637. doi: 10.1002/adma201301804

Rourke, J. P., Pandey, P. A., Moore, J. J., Bates, M., Kinloch, I. A., Young, R. J., et al. (2011). The real graphene oxide revealed: stripping the oxidative debris from the graphene-like sheets. Angew. Chem. Int. Ed. Engl. 50, 3173-3177. doi: 10.1002/anie.201007520

Ruiz, O. N., Fernando, K. A. S., Wang, B., Brown, N. A., Luo, P. G., McNamara, N. D., et al. (2011). Graphene oxide: a nonspecific enhancer of cellular growth. ACS Nano 5, 8100-8107. doi: 10.1021/nn202699t

Saburi, E., Islami, M., Hosseinzadeh, S., Moghadam, A. S., Mansour, R. N., Azadian, E., et al. (2019). In vitro osteogenic differentiation potential of the human induced pluripotent stem cells augments when grown on Graphene oxide-modified nanofibers. Gene 696, 72-79. doi: 10.1016/j.gene. 2019.02.028

Sánchez-González, S., Diban, N., Bianchi, F., Ye, H., and Urtiaga, A. (2018). Evidences of the effect of GO and rGO in PCL membranes on the differentiation and maturation of human neural progenitor cells. Macromol. Biosci. 18:e1800195. doi: 10.1002/mabi.201800195

Sasidharan, A., Panchakarla, L. S., Chandran, P., Menon, D., Nair, S., Rao, C. N. R., et al. (2011). Differential nano-bio interactions and toxicity effects of pristine versus functionalized graphene. Nanoscale 3, 2461-2464. doi: 10.1039/clnr10172b

Seabra, A. B., Paula, A. J., de Lima, R., Alves, O. L., and Durán, N. (2014). Nanotoxicity of graphene and graphene oxide. Chem. Res. Toxicol. 27, 159-168. doi: 10.1021/tx400385x

Shih, C.-J., Vijayaraghavan, A., Krishnan, R., Sharma, R., Han, J.-H., Ham, M.-H., et al. (2011). Bi- and trilayer graphene solutions. Nat. Nanotechnol. 6, 439-445. doi: 10.1038/nnano.2011.94

Skocek, O., Nöbauer, T., Weilguny, L., Martínez Traub, F., Xia, C. N., Molodtsov, M. I., et al. (2018). High-speed volumetric imaging of neuronal activity in freely moving rodents. Nat. Methods 15, 429-432. doi: 10.1038/s41592-018-0008-0

Stankovich, S., Dikin, D. A., Piner, R. D., Kohlhaas, K. A., Kleinhammes, A., Jia, Y., et al. (2007). Synthesis of graphene-based nanosheets via chemical reduction of exfoliated graphite oxide. Carbon 45, 1558-1565. doi: 10.1016/j.carbon.2007. 02.034

Su, Y., Ping, X., Yu, K. J., Lee, J. W., Fan, J. A., Wang, B., et al. (2017). Inplane deformation mechanics for highly stretchable electronics. Adv. Mater. 29:1604989. doi: 10.1002/adma.201604989

Su, C.-Y., Xu, Y., Zhang, W., Zhao, J., Tang, X., Tsai, C.-H., et al. (2009). Electrical and spectroscopic characterizations of ultra-large reduced graphene oxide monolayers. Chem. Mater. 21, 5674-5680. doi: 10.1021/cm90 $2182 y$

Suk, J. W., Kitt, A., Magnuson, C. W., Hao, Y., Ahmed, S., An, J., et al. (2011). Transfer of CVD-grown monolayer graphene onto arbitrary substrates. ACS Nano 5, 6916-6924. doi: 10.1021/nn201207c

Suk, J. W., Lee, W. H., Lee, J., Chou, H., Piner, R. D., Hao, Y., et al. (2013). Enhancement of the electrical properties of graphene grown by chemical vapor deposition via controlling the effects of polymer residue. Nano Lett. 13, 1462-1467. doi: 10.1021/nl304420b

Sun, X., Liu, Z., Welsher, K., Robinson, J. T., Goodwin, A., Zaric, S., et al. (2008). Nano-graphene oxide for cellular imaging and drug delivery. Nano Res. 1, 203-212. doi: 10.1007/s12274-008-8021-8

Tang, M., Song, Q., Li, N., Jiang, Z., Huang, R., and Cheng, G. (2013). Enhancement of electrical signaling in neural networks on graphene films. Biomaterials 34, 6402-6411. doi: 10.1016/j.biomaterials.2013.05.024

Taylor, I. M., Robbins, E. M., Catt, K. A., Cody, P. A., Happe, C. L., and Cui, X. T. (2017). Enhanced dopamine detection sensitivity by PEDOT/graphene oxide coating on in vivo carbon fiber electrodes. Biosens. Bioelectron. 89, 400-410. doi: 10.1016/j.bios.2016.05.084

Thunemann, M., Lu, Y., Liu, X., Kílíç, K., Desjardins, M., Vandenberghe, M., et al. (2018). Deep 2-photon imaging and artifact-free optogenetics through transparent graphene microelectrode arrays. Nat. Commun. 9:2035. doi: 10.1038/s41467-018-04457-5

Titov, A. V., Král, P., and Pearson, R. (2010). Sandwiched graphene-membrane superstructures. ACS Nano 4, 229-234. doi: 10.1021/nn9015778
Tu, Q., Pang, L., Chen, Y., Zhang, Y., Zhang, R., Lu, B., et al. (2014). Effects of surface charges of graphene oxide on neuronal outgrowth and branching. Analyst 139, 105-115. doi: 10.1039/c3an01796f

Tu, Y., Lv, M., Xiu, P., Huynh, T., Zhang, M., Castelli, M., et al. (2013). Destructive extraction of phospholipids from Escherichia coli membranes by graphene nanosheets. Nat. Nanotechnol. 8, 594-601. doi: 10.1038/nnano.2013.125

Tu, Z., Wycisk, V., Cheng, C., Chen, W., Adeli, M., and Haag, R. (2017). Functionalized graphene sheets for intracellular controlled release of therapeutic agents. Nanoscale 9, 18931-18939. doi: 10.1039/c7nr 06588d

Varshney, L. R., Chen, B. L., Paniagua, E., Hall, D. H., and Chklovskii, D. B. (2011). Structural properties of the caenorhabditis elegans neuronal network. PLoS Comput. Biol. 7:e1001066. doi: 10.1371/journal.pcbi. 1001066

Veliev, F., Briançon-Marjollet, A., Bouchiat, V., and Delacour, C. (2016). Impact of crystalline quality on neuronal affinity of pristine graphene. Biomaterials 86 , 33-41. doi: 10.1016/j.biomaterials.2016.01.042

Voiry, D., Yang, J., Kupferberg, J., Fullon, R., Lee, C., Jeong, H. Y., et al. (2016). High-quality graphene via microwave reduction of solutionexfoliated graphene oxide. Science 353, 1413-1416. doi: 10.1126/science. aah3398

Wang, S., Ang, P. K., Wang, Z., Tang, A. L. L., Thong, J. T. L., and Loh, K. P. (2010). High mobility, printable, and solution-processed graphene electronics. Nano Lett. 10, 92-98. doi: 10.1021/nl9028736

Wang, L., Boutilier, M. S. H., Kidambi, P. R., Jang, D., Hadjiconstantinou, N. G., and Karnik, R. (2017). Fundamental transport mechanisms, fabrication and potential applications of nanoporous atomically thin membranes. Nat. Nanotechnol. 12, 509-522. doi: 10.1038/nnano.2017.72

Wang, S., Chia, P.-J., Chua, L.-L., Zhao, L.-H., Png, R.-Q., Sivaramakrishnan, S., et al. (2008). Band-like transport in surface-functionalized highly solution-processable graphene nanosheets. Adv. Mater. 20, 3440-3446. doi: 10.1002/adma.200800279

Wang, J., Cui, C., Nan, H., Yu, Y., Xiao, Y., Poon, E., et al. (2017). Graphene sheet-induced global maturation of cardiomyocytes derived from human induced pluripotent stem cells. ACS Appl. Mater. Interfaces 9, 25929-25940. doi: 10.1021/acsami.7b08777

Wang, Y., Li, Z., Hu, D., Lin, C.-T., Li, J., and Lin, Y. (2010). Aptamer/graphene oxide nanocomplex for in situ molecular probing in living cells. J. Am. Chem. Soc. 132, 9274-9276. doi: 10.1021/ja103169v

Wang, F., Liu, B., Ip, A. C. F., and Liu, J. (2013). Orthogonal adsorption onto nano-graphene oxide using different intermolecular forces for multiplexed delivery. Adv. Mater. 25, 4087-4092. doi: 10.1002/adma.2013 01183

Wang, Y., Wang, K., Zhao, J., Liu, X., Bu, J., Yan, X., et al. (2013). Multifunctional mesoporous silica-coated graphene nanosheet used for chemo-photothermal synergistic targeted therapy of glioma. J. Am. Chem. Soc. 135, 4799-4804. doi: $10.1021 /$ ja312221g

Weaver, C. L., LaRosa, J. M., Luo, X., and Cui, X. T. (2014). Electrically controlled drug delivery from graphene oxide nanocomposite films. ACS Nano 8, 1834-1843. doi: 10.1021/nn406223e

Welsher, K., Liu, Z., Daranciang, D., and Dai, H. (2008). Selective probing and imaging of cells with single walled carbon nanotubes as near-infrared fluorescent molecules. Nano Lett. 8, 586-590. doi: 10.1021/nl072949q

Wilks, S. J., Richardson-Burns, S. M., Hendricks, J. L., Martin, D. C., and Otto, K. J. (2009). Poly(3,4-ethylenedioxythiophene) as a micro-neural interface material for electrostimulation. Front. Neuroeng. 2:7. doi: 10.3389/neuro.16. 007.2009

Won, S., Hwangbo, Y., Lee, S.-K., Kim, K.-S., Kim, K.-S., Lee, S.-M., et al. (2014). Double-layer CVD graphene as stretchable transparent electrodes. Nanoscale 6, 6057-6064. doi: 10.1039/c4nr00265b

Xu, Y., Wu, Q., Sun, Y., Bai, H., and Shi, G. (2010). Three-dimensional self-assembly of graphene oxide and DNA into multifunctional hydrogels. ACS Nano 4, 7358-7362. doi: 10.1021/nn1027104

Yang, H.-W., Hua, M.-Y., Hwang, T.-L., Lin, K.-J., Huang, C.-Y., Tsai, R.-Y., et al. (2013). Non-invasive synergistic treatment of brain tumors by targeted chemotherapeutic delivery and amplified focused ultrasoundhyperthermia using magnetic nanographene oxide. Adv. Mater. 25, 3605-3611. doi: 10.1002/adma.201301046 
Yang, X., Tu, Y., Li, L., Shang, S., and Tao, X.-M. (2010). Well-dispersed chitosan/graphene oxide nanocomposites. ACS Appl. Mater. Interfaces 2, 1707-1713. doi: 10.1021/am100222m

Yi, H., Wu, L.-Q., Bentley, W. E., Ghodssi, R., Rubloff, G. W., Culver, J. N., et al. (2005). Biofabrication with chitosan. Biomacromolecules 6, 2881-2894. doi: 10.1021/bm0504101

Yoo, J., Kim, J., Baek, S., Park, Y., Im, H., and Kim, J. (2014). Cell reprogramming into the pluripotent state using graphene based substrates. Biomaterials 35, 8321-8329. doi: 10.1016/j.biomaterials.2014. 05.096

Yu, K. J., Yan, Z., Han, M., and Rogers, J. A. (2017). Inorganic semiconducting materials for flexible and stretchable electronics. NPJ Flex. Electron. 1:4. doi: 10.1038/s41528-0170003-z

Zanni, E., De Bellis, G., Bracciale, M. P., Broggi, A., Santarelli, M. L., Sarto, M. S., et al. (2012). Graphite nanoplatelets and Caenorhabditis elegans: insights from an in vivo model. Nano Lett. 12, 2740-2744. doi: 10.1021/n $1204388 \mathrm{p}$

Zhang, Y., Ali, S. F., Dervishi, E., Xu, Y., Li, Z., Casciano, D., et al. (2010). Cytotoxicity effects of graphene and single-wall carbon nanotubes in neural phaeochromocytoma-derived PC12 cells. ACS Nano 4, 3181-3186. doi: $10.1021 / \mathrm{nn} 1007176$

Zhang, S., Yang, K., Feng, L., and Liu, Z. (2011). in vitro and in vivo behaviors of dextran functionalized graphene. Carbon 49, 4040-4049. doi: 10.1016/j.carbon. 2011.05.056

Zhang, X., Yin, J., Peng, C., Hu, W., Zhu, Z., Li, W., et al. (2011). Distribution and biocompatibility studies of graphene oxide in mice after intravenous administration. Carbon 49, 986-995. doi: 10.1016/j.carbon.2010.11.005

Conflict of Interest Statement: The authors declare that the research was conducted in the absence of any commercial or financial relationships that could be construed as a potential conflict of interest.

Copyright (c) 2019 Kitko and Zhang. This is an open-access article distributed under the terms of the Creative Commons Attribution License (CC BY). The use, distribution or reproduction in other forums is permitted, provided the original author(s) and the copyright owner(s) are credited and that the original publication in this journal is cited, in accordance with accepted academic practice. No use, distribution or reproduction is permitted which does not comply with these terms. 